Document downloaded from:

http://hdl.handle.net/10251/61404

This paper must be cited as:

Gaona Cordero, A.; Moreno, JM.; Velty, A.; Díaz Morales, UM.; Corma Canós, A. (2014). One-pot synthesis of hierarchical porous layered hybrid materials based on aluminosilicate sheets and organic functional pillars. Journal of Materials Chemistry A. 2(45):19360-19375. doi:10.1039/c4ta04742g.

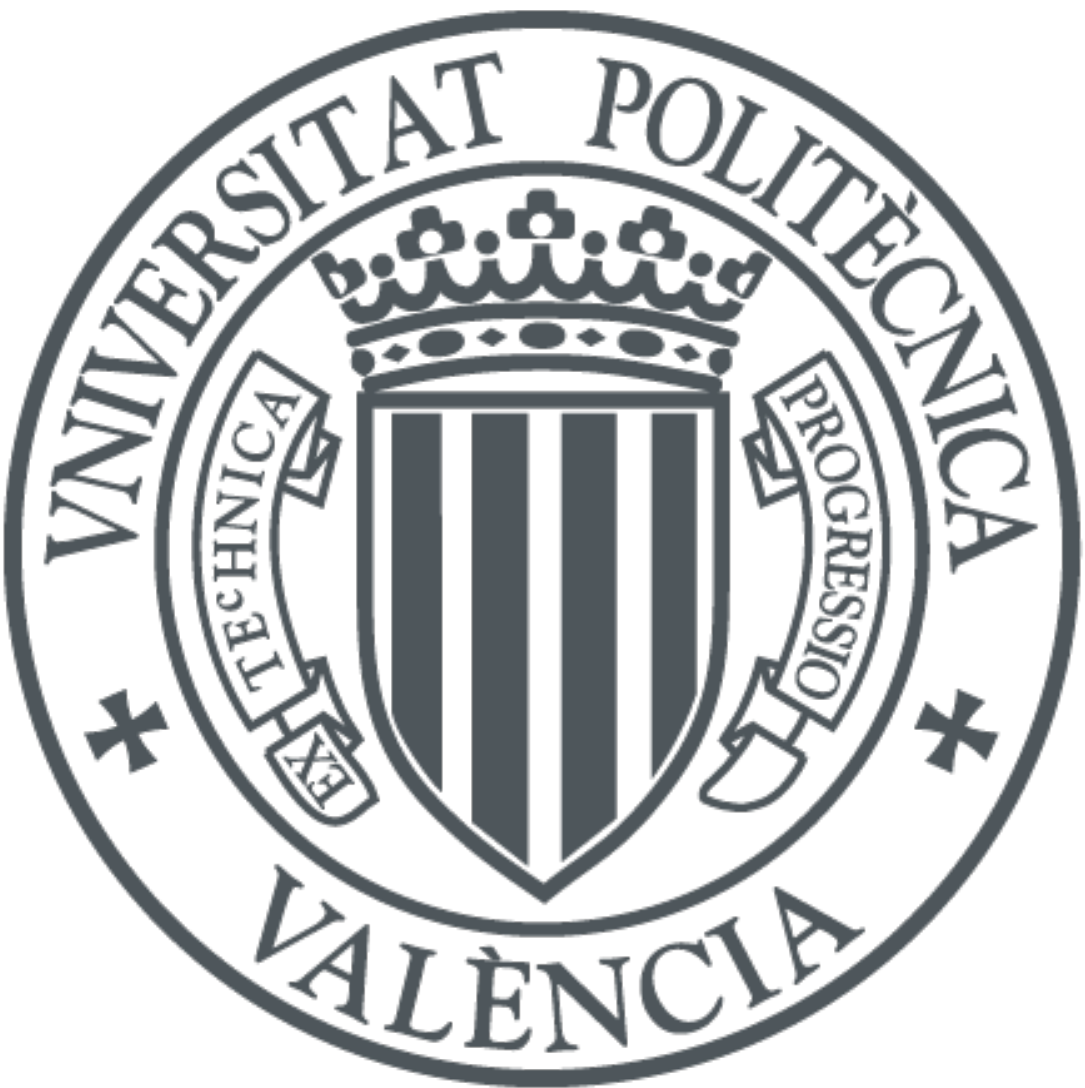

The final publication is available at

http://dx.doi.org/10.1039/c4ta04742g

Copyright Royal Society of Chemistry

Additional Information 


\title{
One-pot synthesis of hierarchical porous layered hybrid materials based on aluminosilicate sheets and organic functional pillars
}

\author{
Aidé Gaona, José María Moreno, Alexandra Velty, Urbano Díaz*, Avelino Corma*
}

Instituto de Tecnología Química, UPV-CSIC, Universidad Politécnica de Valencia, Avenida de los Naranjos s/n, E-46022 Valencia, Spain

Tel.: +34963877800 FAX: +34963877809 E-mail: acorma@itq.upv.es; udiaz@itq.upv.es

\begin{abstract}
Layered hybrid materials (LHMs) based on ordered silicoaluminate sheets linked with organic fragments, perpendicularly located and stabilized in the interlayer space, were synthesized by one-pot direct hydrothermal process, in absence of structural directing agents (SDAs), and using bridged silsesquioxanes as organosilicon precursors. By following the synthesis described here, the preliminary preparation of inorganic layered precursors, post-synthesis swelling and/or pillaring treatments can be avoided. The physico-chemical and structural characteristics of the materials were studied by chemical and thermogravimetrical analyses, X-ray diffraction, TEM microscopy, spectroscopic techniques (NMR and FTIR) and textural measurements. The complete exchange of intracrystalline sodium cations by protons, without substantial structural alteration of hybrid materials, facilitated the generation of hybrid materials, which contained acid and base sites located in the inorganic (silicoaluminate layers) and in the organic interlayer linkers, respectively, being the resultant acid-base materials active and selective catalysts.
\end{abstract}

Keywords: Layered hybrid materials; Silicoaluminates; Hydrothermal synthesis; Organic linkers; Acid-base materials; C-C bond forming reactions. 


\section{Introduction}

The preparation of new type of hierarchically structured hybrid materials, in which organic and inorganic properties merge, is a topic within continuous expansion. ${ }^{1,2}$ The possibility to generate materials that combine at nanometric scale the main advantages of inorganic solids (hydrothermal, structural and mechanical stability) together with those exhibited by organic compounds (as flexibility and functionality), allows their use from catalysis to sensoring, adsorption, photoluminescence, magnetism or electronics. ${ }^{3}$ The use, during the synthesis process, of suitable precursors in which organic and inorganic building blocks are previously connected is decisive to obtain stable, homogeneous and hierarchically structured Class II hybrid materials (strong coordinative or covalent interaction between the different structural fragments), ${ }^{4}$ avoiding phase's separation between the different builders forming their network. ${ }^{5,6}$ Specifically, bridged silsesquioxanes, ${ }_{3}\left(\mathrm{R}^{\prime} \mathrm{O}\right) \mathrm{Si}-\mathrm{R}-\mathrm{Si}\left(\mathrm{OR}^{\prime}\right)_{3}$, and organometallic compounds have been the most relevant organic-inorganic precursors used to obtain periodic mesoporous organosilicas (PMOs) or metalorganic frameworks (MOFs), respectively. ${ }^{7,8}$ The preparation of these types of hybrid solids by sol-gel, hydrothermal or solvothermal methods" ${ }^{10}$ is accompanied by the in situ formation of inorganic builders, such as silicon tetrahedra or metallic nodes, which facilitate the final assembly of the organic and inorganic fragments through covalent or coordination bonds.

Within the family of hybrid materials, the lamellar solids occupy a relevant place, owing to the versatility offered by layered precursors which allow the controlled modification of the interlayer space between the ordered inorganic sheets. ${ }^{11,12}$ Several lamellar precursors with different physico-chemical properties, such as clays, layered double hydroxides, titanates, phosphates, graphites, silicates or zeolites, have successfully been employed to generate organic-inorganic materials or nanocomposites with different functionalities and properties. ${ }^{13}$, ${ }^{14}$ The simplest methodology employed to synthesize layered hybrid materials has been the inclusion of organic molecules in the interlayer space by either adsorption or ion exchange methods. ${ }^{15}$ In the latter case, Class I hybrid solids were obtained by the weaker electrostatic interactions (such as Van der Waals or hydrogen bonding) between organic compounds and inorganic sheets, ${ }^{4,16}$ being appreciable the leaching of the organic component and the partial blockage of the interlayer galleries by an excess of organic moieties, with the corresponding negative impact on diffusion, adsorption and reactivity of molecules (Figure S1). ${ }^{17}$

On the other hand, the direct assembly between organic compounds and inorganic layers has allowed the generation of stable, robust and irreversible Class II hybrid materials 
with different functionalities isolated into their regular structure. ${ }^{18}$ This is the case of hybrid materials derived from delaminated zeolites, such as ITQ-2 and ITQ-6, in which specific organocatalysts, containing acid, base or chiral centers, are covalently supported onto MWW or ferrieritic individual layers. ${ }^{19,20,21}$ Another possibility has been the incorporation, as stable pillars, of organic functional fragments in the interlayer space, generating ordered hybrid materials with high reactant accessibility owing to the additional mesoporous galleries created between the inorganic zeolitic sheets (Figure S1). These approaches have been very effective to heterogenize different homogeneous catalysts without appreciable activity loss. ${ }^{22,}{ }^{23}$ Those methodologies were validated by the recent preparation of multifunctional zeolitic hybrid materials. Specifically, bi-functional organic-inorganic layered materials were synthesized by the intercalation of arylic silsesquioxane molecules between inorganic zeolitic MWW layers. ${ }^{24}$ The organic linkers were conformed by two condensed silyl-arylic groups from disilane molecules, which reacted with the external silanol groups of the zeolitic layers. The resultant hybrids exhibited micropores within the inorganic MWW layers together with a well-defined mesoporous system delimited by the organic linkers and the zeolitic sheets. Final amination by a post-synthesis treatments introduced base groups in the organic linkers, located near the acid sites present in the structural inorganic zeolitic counterpart. Through this methodology, it has been possible to prepare acid-base catalysts where the acid sites are of zeolitic nature and the base sites are part of the organic builders. These hybrid materials acted successfully as bifunctional catalysts for performing a two-step cascade reactions that involved the catalytic conversion of benzaldehyde dimethylacetal into benzylidene malononitrile, using only one recoverable catalyst. ${ }^{25,26}$ Similar approaches have recently been followed to obtain hierarchical layered hybrid materials with tunable textural properties by pillaring IPC-1P precursor, prepared from modified Ge-zeolite UTL, with several bridged silsesquioxanes or polyhedral oligomeric siloxanes. ${ }^{27}$

Despite the high potentiality and specificity of the lamellar hybrid materials prepared through pillarization with layered inorganic precursors and bridged silsesquioxanes as intercalation agents, the synthesis of the hybrid materials involves a series of complex steps to obtain the final product. In most of the cases, the steps required are: a) synthesis of the layered inorganic precursor; b) swelling and pillarization of starting layered precursors; c) extraction and post-functionalization processes. ${ }^{28,29,30,31}$ As can be seen, the multi-step synthesis method involves a long route that, on top, requires recovery and isolation of intermediate lamellar hybrid solids. Moreover, it is difficult to avoid an inhomogeneous distribution of organic pillars located in the interlayer space, which could be a problem during 
the catalytic reactions. ${ }^{32}$ There is no doubt that it would be of much interest to synthesize ordered, regular and stable lamellar organic-inorganic hybrid materials in only one step. For doing that, Alauzun et al. and Moreau et al. attempted to synthesize directly lamellar hybrid materials, using bridged silsesquioxanes (disilanes) during acid or base sol-gel synthesis processes. ${ }^{33,34}$ The results were overall satisfactory when the organic linkers were formed by 10-20 $-\mathrm{CH}_{2}-$ units or contained ureido-phenyl groups, because hydrogen bonds' interactions between organic moieties were strongly favored. The resultant materials were structured by silica layers, generated by polymerization of terminal siloxane groups present in the disilane precursors, being they separated by the organic linkers. However, the elevated number of organic fragments located in the interlayer space resulted in the preparation of hybrid materials without porosity and completely collapsed, minimizing their potential use as solid catalysts. ${ }^{35}$

Recently, Bellussi et al. have made important advances in this topic by reporting a new family of layered hybrid organic-inorganic materials, named ECS (Eni Carbon Silicates). They are synthesized in only one step, using rigid arylic bridged silsesquioxanes, alkaline medium and sodium aluminate as additional sources ( $\mathrm{Si} / \mathrm{Al} \sim 1$ ), within hydrothermal conditions and in absence of structure directing agents. ${ }^{36,37,38}$ In this way, a new generation of crystalline hybrid aluminosilicates with, in some cases, open porosity (Figure S2) and conformed by inorganic layers structured from $\left[\mathrm{AlO}_{4}\right]$ and $\left[\mathrm{SiO}_{3} \mathrm{C}\right]$ tetrahedron, which are separated by aryilic bridges, have been obtained. ${ }^{39}$ The crystal structure determination has shown that an elevated number of these lamellar materials contain a double porous system: intra-layered conformed by microporous channels normally delimited by six member rings (6MR), and an inter-layered space owing to the galleries generated between inorganic sheets and rigid aromatic linkers. In this way, layered hybrid materials with specific surface areas and porous volumes, sometimes, close to $350 \mathrm{~m} \mathrm{~g}^{2} \mathrm{~g}^{-1}$ and $0.70 \mathrm{~cm}^{3} \mathrm{~g}^{-1}$ were obtained. ${ }^{40,}{ }^{41}$ Even, layered ECS-type materials with inorganic sheets built from only three-ring secondary building units have recently been described ${ }^{42}$ However, these interesting materials have only been successfully prepared, as far as we know, with rigid and non-catalytically active arylic fragments intercalated in the interlayer space.

In the present work, we have succeeded in the preparation of several ordered layered hybrid materials (LHM) by one-pot structure directing free synthesis. They are conformed by aluminosilicate sheets, related with ECS-type family above commented, but separated and covalently connected between them by flexible and active bridged silsesquioxanes which 
contain stabilized functional ethylendiamino groups as organic linkers. During the direct onestep hydrothermal synthesis, we have studied the influence of the synthesis variables in order to prepare lamellar homogeneous porous materials with more accessible active organic and inorganic functions. Following the method here described, it is possible to avoid a multi-step synthesis involving swelling, pillarization and extraction post-synthesis treatments. The high exchange capacity of these layered hybrid materials was confirmed through the complete substitution of the intracrystalline compensation cationic sodium by acid protons, allowing the generation of functional hybrid catalysts which contain acid and base sites located in the inorganic sheets and in the interlayer organic linkers, respectively, being these latter active for $\mathrm{C}-\mathrm{C}$ bond forming reactions. The stability of the hybrid lamellar materials has been confirmed through the different catalytic reuses without observing appreciable activity loss. 


\section{Experimental}

\section{Reagents}

Layered hybrid materials (LHM) and ECS-1 materials were synthesized using bis(trimethoxysilylpropyl)ethylendiamino (BTMN, Abcr), triethoxymethylsilane (TEMS, Aldrich), 1,4-bis-(triethoxysilyl)-benzene (BTEB, Abcr) and tetraethyl orthosilicate (TEOS, Aldrich) as silica sources. Sodium aluminate $\left(\mathrm{NaAlO}_{2}, \mathrm{Carlo}\right.$ Erba) and $\mathrm{NaOH}$ (Aldrich) were used as aluminum and alkaline sources, respectively. Diluted $0.1 \mathrm{M} \mathrm{HCl}$ solution was used during exchange treatments.

\section{Synthesis of Layered Hybrid Materials}

\section{LHM-BTMN and LHM-BTMN-H}

The reactant mixture was prepared by drop wise addition of the organosilicon BTMN precursor to an aqueous solution of sodium aluminate $\left(\mathrm{NaAlO}_{2}\right)$ and $\mathrm{NaOH}$, to achieve the following molar ratios: $\mathrm{Si} / \mathrm{Al}=0.55, \mathrm{Na} / \mathrm{Si}=2.30, \mathrm{NaOH} / \mathrm{Si}=0.33$ and $\mathrm{H}_{2} \mathrm{O} / \mathrm{Si}=23.90$. The resulting gel was homogeneized by stirring at room temperature and was charged into a stainless steel autoclave and heated at 135 o $\mathrm{C}$ for 9 days under autogeneous pressure and static conditions. Once cooled to room temperature, the solid product was isolated, washed with distilled water and dried overnight at $60{ }^{\circ} \mathrm{C}$, obtaining the sample named LHM-BTMN.

The sodium cations exchange treatment to obtain the protonic solid (LHM-BTMN-H) was performed by dispersing $1.5 \mathrm{~g}$ of LHM-BTMN in $20 \mathrm{~mL}$ of MilliQ water and slowly dropping an $\mathrm{HCl}$ aqueous solution (0.1 M) under continuous stirring at room temperature up to achieve a constant $\mathrm{pH}$ of 4 . The solution was stirred for additional $48 \mathrm{~h}$ at room temperature, and was filtered and washed with EtOH and distilled water until neutral $\mathrm{pH}$. The sample was overnight dried at $60 \stackrel{\circ}{ } \mathrm{C}$.

\section{LHM-TEMS and LHM-TEMS-H}

The synthesis methodology to obtain LHM-TEMS was the same above described for LHM-BTMN, except that in the LHM-TEMS a mixture of silicon and organosilicon sources was used. Specifically, $50 \%, 25 \%$ and $25 \%$ of silicon moles were incorporated in the synthesis gel as BTMN, TEMS and TEOS, respectively. The post-synthesis acid exchange treatment to prepare LHM-TEMS-H was also the same above described. In this case, before the catalytic tests, the sample LHM-TEMS-H was pretreated by refluxing with an ethanolic solution of ethanolamine ( $20 \% \mathrm{vol})$ for $15 \mathrm{~h}$ at 80 으 $\mathrm{C}$ to neutralize the protonated amino groups formed during the acid exchange treatment. The recovered sample was stirred with pure EtOH for $24 \mathrm{~h}$ 
at room temperature to remove the excess of ethanolamine retained. The solid was filtered and washed with EtOH and distilled water, and finally overnight dried at $60{ }^{\circ} \mathrm{C}$.

\section{ECS-1}

In this case, the methodology followed was similar to above described for LHM-BTMN and LHM-TEMS materials, using BTEB as the only organosilicon source and achieving in the synthesis gel the following molar ratios: $\mathrm{Si} / \mathrm{Al}=1.20, \mathrm{Na} / \mathrm{Si}=1.02, \mathrm{NaOH} / \mathrm{Si}=0.15$ and $\mathrm{H}_{2} \mathrm{O} / \mathrm{Si}=11$, such as it was described in the bibliography. ${ }^{37}$ The hydrothermal synthesis was carried out at $100 \stackrel{\circ}{ }$ for 9 days under static conditions.

\section{Catalytic tests}

Condensation reactions were carried out in a closed conic vessel under nitrogen atmosphere and magnetic stirring.

- Benzylidene malononitrile: A mixture of benzaldehyde $(2.94 \mathrm{mmol})$ and malononitrile $(2.99$ $\mathrm{mmol}$ ) at $30 \stackrel{\circ}{\circ}$, under an inert atmosphere $\left(\mathrm{N}_{2}\right)$, was magnetically stirred, and $13 \mathrm{mg}$ of catalyst, 2.5 mol\% of $\mathrm{N}$, were added. Acetonitrile was used as solvent $(1 \mathrm{~mL})$.

- Ethylcyanocinnamate: A mixture of benzaldehyde $(2.67 \mathrm{mmol})$ and ethyl cyanoacetate $(2.87$ $\mathrm{mmol}$ ) at $60 \stackrel{\circ}{\circ}$, under an inert atmosphere $\left(\mathrm{N}_{2}\right)$, was magnetically stirred, and $25 \mathrm{mg}$ of catalyst, $5 \mathrm{~mol} \%$ of $\mathrm{N}$, were added; $1 \mathrm{~mL}$ of acetonitrile was used as solvent.

- Ethyl 2-benzylideneacetoacetate: A mixture of benzaldehyde $(2.25 \mathrm{mmol})$ and ethyl acetoacetate $(2.01 \mathrm{mmol})$ at $80 \stackrel{\circ}{\circ}$, under an inert atmosphere $\left(\mathrm{N}_{2}\right)$, was magnetically stirred, and $35 \mathrm{mg}$ of catalyst, $10 \mathrm{~mol} \%$ of $\mathrm{N}$, were added; $1 \mathrm{~mL}$ of acetonitrile was used as solvent.

Photostabilizers were synthesized in a two-necked-round bottom flask equipped with a condenser under nitrogen atmosphere and magnetic stirring.

- 2-cyano-3-(4-hydroxy-3,5-dimethoxyphenyl) 2-propenoic acid ethyl ester: A mixture of syringaldehyde $(7.5 \mathrm{mmol})$ and ethyl cyanoacetate $(8.5 \mathrm{mmol}), 3 \mathrm{~mL}$ of dimethylformamide, at $70 \stackrel{\circ}{ } \mathrm{C}$, under an inert atmosphere $\left(\mathrm{N}_{2}\right)$, was magnetically stirred, and $45 \mathrm{mg}$ of catalyst 2.5 mol\% of $\mathrm{N}$, were added.

- Ethyl $\alpha$-cyano-4-hydroxy-3-methoxycinnamate: A mixture of vanillin (2.5 mmol), ethyl cyanoacetate $(2.7 \mathrm{mmol})$ and $1 \mathrm{~mL}$ of acetonitrile at $70 \stackrel{\circ}{\circ}$, under an inert atmosphere $\left(\mathrm{N}_{2}\right)$, was magnetically stirred, and $15 \mathrm{mg}$ of catalyst, $3.4 \mathrm{~mol} \%$ of $\mathrm{N}$, were added.

In all cases, samples were taken periodically during the reaction and analyzed by GC/MS and GC equipped with a HP-5 column $(30 \mathrm{~m} * 0.32 * 0.25 \mu \mathrm{m})$ and a FID as detector. 


\section{Characterization Techniques}

XRD analysis was carried out with a Philips X'PERT diffractometer equipped with a proportional detector and a secondary graphite monochromator. Data were collected stepwise over the $2 \circ \leq 2 \theta \leq 20^{\circ}$ angular region, with steps of $0.02 \circ 2 \theta, 20 \mathrm{~s} / \mathrm{step}$ accumulation time and CuK $\alpha(\lambda=1.54178 \AA$ ) radiation. Transmission electron microscopy (TEM) micrographs were obtained with a JEOL JEM2100F electron microscope operating at $200 \mathrm{keV}$. The samples were prepared directly by dispersing the powders onto carbon copper grids. C, N, S and H contents were determined with a Carlo Erba 1106 elemental analyzer, while Si, Al and Na contents were obtained by means of atomic absorption spectroscopy (Spectra AA 10 Plus, Varian). Thermogravimetric and differential thermal analyses (TGA-DTA) were conducted in an air stream with a Metler Toledo TGA/SDTA 851E analyzer. IR spectra were obtained in a Nicolet 710 spectrometer $\left(4 \mathrm{~cm}^{-1}\right.$ resolution), using a conventional greaseless cell. Wafers of $10 \mathrm{mg} \cdot \mathrm{cm}^{-}$

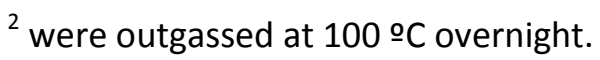

Nitrogen adsorption isotherms were measured at -196 으 with a Micromeritics ASAP 2010 volumetric adsorption analyser. Before the measurements, the samples were outgassed

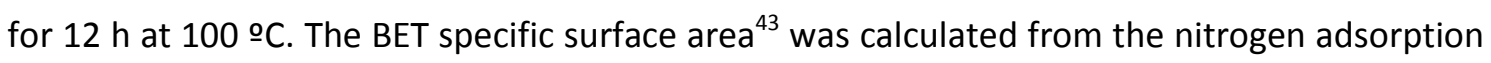
data in the relative pressure range from 0.04 to 0.2 . The total pore volume ${ }^{44}$ was obtained from the amount of $\mathrm{N}_{2}$ adsorbed at a relative pressure of about 0.99. External surface area and micropore volume were estimated using the $t$-plot method in the $t$ range from 3.5 to 5 . The pore diameter and the pore size distribution were calculated using the Barret-Joyner-Halenda (BJH) method ${ }^{45}$ on the adsorption branch of the nitrogen isotherms.

Solid state MAS-NMR spectra were recorded at room temperature under magic angle spinning (MAS) in a Bruker AV-400 spectrometer. The single pulse ${ }^{29} \mathrm{Si}$ spectra were acquired at 79.5 $\mathrm{MHz}$ with a $7 \mathrm{~mm}$ Bruker BL-7 probe using pulses of $3.5 \mu$ s corresponding to a flip angle of $3 / 4 \pi$ radians, and a recycle delay of $240 \mathrm{~s} .{ }^{27} \mathrm{Al}$ spectra were obtained at $104.2 \mathrm{MHz}$ using a 4 $\mathrm{mm}$ Bruker BL-4 probe. Pulses of $0.5 \mu$ s to flip the magnetization $\pi / 20 \mathrm{rad}$, and a recycle delay of $2 \mathrm{~s}$ were used. The ${ }^{1} \mathrm{H}$ to ${ }^{13} \mathrm{C}$ cross-polarization (CP) spectra were acquired by using a $90^{\circ}$ pulse for ${ }^{1} \mathrm{H}$ of $5 \mu \mathrm{s}$, a contact time of $5 \mathrm{~ms}$, and a recycle of $3 \mathrm{~ms}$. The ${ }^{13} \mathrm{C}$ spectra were recorded with a $7 \mathrm{~mm}$ Bruker $\mathrm{BL}-7$ probe and at a sample spinning rate of $5 \mathrm{kHz} .{ }^{13} \mathrm{C},{ }^{29} \mathrm{Si}$ and ${ }^{27} \mathrm{Al}$ were referred to adamantine, tetramethylsilane and an aqueous solution of $\mathrm{Al}\left(\mathrm{NO}_{3}\right)_{3}$, respectively. 


\section{Results and Discussion}

\section{Synthesis and characterization}

Normally, the preparation in alkaline media of synthesis gels with high aluminum content and silicon precursors containing reactive siloxane groups, favors the generation of stable microporous silica-aluminas with low Si/Al molar ratios. ${ }^{46}$ This was also observed in the materials here considered which were formed by ordered inorganic $\mathrm{SiO}_{2}-\mathrm{Al}_{2} \mathrm{O}_{3}$ sheets, separated by organic spacers located in the interlayer space, coming from bridged silsesquioxanes used as organosilicon precursors. The electrostatic interaction established between the organic linkers favored the formation of ordered silicoaluminate sheets which are perpendicularly aligned to the interlayered organic spacers (Figure 1). It is important to point out that the crystallization was achieved in absence of structure directing agents in only one hydrothermal step, avoiding swelling, pillarization and extraction post-synthesis treatments of the previously synthesized inorganic layered precursors. ${ }^{47}$

The XRD patterns obtained for the as-synthesized samples showed the existence of first typical (100) diffraction band characteristic of ordered layered materials, being possible to accurately estimate the basal space (Figure 2). Specifically, the hybrid material prepared in presence of disilanes containing arylic bridges (BTEB), ${ }^{37}$ exhibited a basal space of $11.9 \AA$ which is in agreement with the presence of rigid arylic fragments with $6.5 \AA$ of length located in the interlayer space (Figure 2a). Taking that into account, then the silicoaluminate sheet's thickness should be approximately $5.4 \AA$. This data would be in agreement with similar thin inorganic sheets, as for instance makatite-type, which are forming conventional inorganic layered silicates such as magadiite or kenyaite, ${ }^{48,}{ }^{49}$ and silicoaluminates obtained from synthesis gels with high aluminum content. ${ }^{50}$ Probably, these types of thin sheets are structured by effective covalent connection between silicon and aluminum tetrahedron, forming intralayer six member rings (6MR) which are along the plane $b c$ of the inorganic layers, such as it was indicated for ECS-type materials. ${ }^{40}$ Instracrystalline sodium cations should be located into these intralayer 6MRs, compensating the positive electric charge due to the presence of aluminum framework. Nevertheless, the location of sodium cations at interlayer space, framework defects and end-edges or corners could also be possible (Figure 3 ).

In the case of materials synthesized in presence of bis-silylated ethylendiamino precursors (LHM-BTMN), the diffraction pattern showed that the inorganic layers were separated by $\sim 11.3 \AA$. If one assumes that the inorganic layers' thickness maybe also around $5.4 \AA$ (Figure 2b), and taking account that the molecular length of the organic fragment from 
BTMN monomers is $\sim 14.1 \AA$, it has to be concluded that partial torsion or inclination of ethylendiamino units in the interlayer space has occurred during the structuration process. In general, the results would indicate the presence of ordered silicoalumina layers, disposed perpendicularly to axis $a$ and separated by homogeneously distributed organic fragments from bridged silsesquioxanes used as organosilicon precursors.

Considering the traditional exchange capacity of porous silicoaluminate materials, associated to their positive charge deficiency, we replaced the cationic sodium species by acidic $\mathrm{H}^{+}$protonic cations that will compensate the negative charge generated by aluminum tetrahedrally coordinated into the framework of inorganic layers (LHM-BTMN-H). This exchange process did not produce a marked shifting of the (100) diffraction band, as compared with the as-synthesized sample, indicating that the lamellar organization is preserved when $\mathrm{Na}^{+}$ions were replaced by protonic species. This behavior shows the stability of the organic linkers which remain unaltered even after the acid exchange treatments (Figure 2c). However, a decrease in the intensity and an increase in the broading of the (100) diffraction band was observed in LHM-BTMN-H material, which can be associated to crystallinity and homogeneity losses, probably due to the acid conditions used during the protonic exchange process. The broad diffraction band observed between 20-25 $2 \theta$ degrees would also be in consonance with the partial generation of amorphous species after the acid exchange treatments.

On the other hand, the layered organic-inorganic materials obtained combining tetraethylorthosilicate (TEOS), bis(trimethoxysilyl)ethylendiamino (BTMN) and triethoxymethylsilane (TEMS) precursors in the synthesis gel (LHM-TEMS), that was done to increase the accessibility of the solids due to the reduction of organic linkers concentration located in the interlayer space, showed a XRD pattern similar to samples obtained only using bridged silsesquioxanes with ethylendiamino (BTMN) or arylic fragments (BTEB) as organosilicon sources, exhibiting the characteristic (100) diffraction band of ordered lamellar materials. However, this band was shifted to lower angles corresponding to basal spaces of $18.1 \AA$ (Figure $2 \mathrm{~d}$ ). This confirmed, that although a lower number of ethylendiamino bridges was intercalated between the silicoalumina layers, the structural order achieved in the hybrid materials was similar to the solids synthesized using only BTMN as precursor. It could also be deduced that the ethylendiamino fragments in LHM-TEMS case are located more vertically than in the materials synthesized with only BTMN silsesquioxane precursors because the interlayer space $(\sim 12.7 \AA)$ is closer to molecular size of organic linkers from disilane precursor (14.1 $\AA$ ). So, the similarity between the molecular lengths of the BTMN organic units and the 
distance between the inorganic layers, estimated from XRD patterns, allowed deducing the effective presence of perpendicularly located organic builders in the interlayer space.

In LHM-TEMS materials, after the protonic exchange, a visible reduction of the intensity of the diffractions bands was also observed, being this effect even more marked than for the exchanged layered materials obtained from LHM-BTMN (Figure 2c). This can be related to the acid conditions employed during the replacement of $\mathrm{Na}^{+}$with $\mathrm{H}^{+}$cations (Figure 2e). In this case, the reduced number of ethylendiamino units placed in the interlayer space, linking the inorganic sheets through covalent interactions, would imply a lower structural stability of the hybrid lamellar network that would be more affected by the acid exchange processes.

It is remarkable that the $(0 \mathrm{kl})$ diffraction bands, observed at higher $2 \theta$ angles than 10 , which are not involved in the lamellar order, were characteristic of each hybrid material, showing that the use of different bridged silsesquioxane precursors or the combination of different organosilicon sources, favors the crystallization of silicoaluminate sheets with similar thickness but with slight structural modifications occurred during the hydrothermal alkaline synthesis (Figure 2). However, in general, the low intensity of the $(0 \mathrm{kl})$ diffraction bands associated to the framework of the sheets in the $b c$ plane would be indicative of the low crystallinity level achieved in this type of silicoaluminate layers, being this fact also observed in standard layered silicates, such as magadiite, kenyaite or illerite, ${ }^{51}$ or in some of the reported ECS-type silicoaluminates. ${ }^{37}$

The lamellar organization of the organic-inorganic materials here synthesized has been evaluated by XRD, being also possible to confirm this morphological spatial organization from transmission electronic microscopy (TEM). The hybrid materials synthesized in presence of BTMN disilane precursors (LHM-BTMN and LHM-TEMS) were formed by individual crystals with plate-type morphology, characteristic of conventional inorganic layered precursors such as lamellar zeolites or silicates (Figure $4 a),{ }^{52}$ and different ordered piled sheets can be observed in each crystal. More specifically, individual silicoaluminate layers were identified and the interlayer space between two contiguous inorganic layers separated by, presumably organic linkers, can be observed (Figure $4 b, c$ and d).

The chemical analyses of the different layered hybrid materials were carried out and the results are shown in Table 1. The results indicate that organic moieties are present in the solids synthesized from organosilane precursors. In the case of materials prepared using only ethylendiamino bridged disilane precursors (LHM-BTMN), the organic content was higher than in the hybrids LHM-TEMS obtained joining both disilanes, BTMN, and monosilane monomers, 
TEMS and TEOS, due to lower organic contribution from the starting precursors in the latter case ( 33\% for LHM-BTMN front $\sim 20 \%$ for LHM-TEMS materials). It is important to point out that the $\mathrm{C} / \mathrm{N}$ molar ratio obtained for LHM-BTMN lamellar materials was close to 4.0 , this being the theoretical value for the propylethylendiamino linkers included in the initial BTMN disilane. This fact would indicate that the organic bridges, which are located between aluminosilicate layers, were preserved during the hydrothermal synthesis process. The lamellar organic-inorganic LHM-TEMS materials, obtained in presence of different organosilanes, exhibited $\mathrm{C} / \mathrm{N}$ molar ratios slightly higher than 4.0, probably because in the synthesis gel a lower content of amino groups, from BTMN, and a higher carbon content, from TEMS precursors, was employed (Table 1).

On the other hand, the chemical analyses obtained corroborate that the acid exchange, performed to replace sodium with proton cations, was completely effective since the sodium content in the final exchanged materials (LHM-BTMN-H and LHM-TEMS-H) was below detection limits (Table 1). Moreover, although the hybrid samples were treated in acid conditions, the rest of their chemical composition remained practically the same, confirming that the chemical characteristics of the organic-inorganic materials after the acid exchange post-synthesis treatments were preserved. This fact was also corroborated from X-ray diffractograms of the exchanged layered hybrid samples, as it was observed above (Figure 2). Additionally, the presence and number of ethylendiamino groups in the exchanged samples were analyzed by direct acid-base titration of the solids (see Supplementary Information), being confirmed the nitrogen content obtained from chemical analyses (Table 1) which oscillated between 3.0 and $5.0 \mathrm{mmol} \mathrm{N} \cdot \mathrm{g}^{-1}$ for the LHM-TEMS-H and LHM-BTMN-H samples, respectively.

Figure S3 shows TGAs and DTAs curves for the as-synthesized layered hybrid materials containing arylic (ECS-1), ethyelendiamino (LHM-BTMN) or LHM-BTMN combined with attached methyl groups (LHM-TEMS). The results from Figure S3 allow establishing, not only the amount of organic fragments incorporated in the lamellar solids, but also their hydrothermal stability. In all cases, besides the hydration water (first weight loss), it is possible to appreciate (Figure S3) a main weight loss assigned to the presence of organic fragments from the bridged silsesquioxanes (BTEB or BTMN) and monosilanes (TEMS) used during the synthesis process. Moreover, the organic content calculated from the thermogravimetric curves was similar to the value obtained by CHN elemental analyses (Table 1), confirming the presence of organic components in the final layered hybrid materials. Nevertheless, a slightly 
higher organic contribution was obtained from thermogravimetrical than from elemental analysis due to the dehydroxylation water generation at elevated temperatures $(\sim 650 \stackrel{\circ}{ })$, attributed to intracrystalline silanol groups condensation, which is not completely estimated from CHN measurements. Additionally, from the DTA curves (Figure S3), it was possible to estimate the thermal stability of the different organic linkers present in the hybrid organicinorganic solids. It can be observed that benzene groups are the most stable, decomposing at temperatures in the $400-650 \stackrel{\circ}{\circ}$ range (ECS-1-type materials). On the other hand, ethylendiamine units exhibited a lower hydrothermal stability $(300-450 \circ \mathrm{C})$.

Similar organic content was also observed in the samples obtained after the acid exchange (LHM-BTMN-H and LHM-TEMS-H), confirming that this post-synthesis treatment did not imply the removing of the organic fragments intercalated in the interlayer space. From the values obtained for the organic content, it was possible to confirm that the practical totality of arylic or ethylendiamino groups present in the synthesis gels were finally incorporated in the materials, the final yield in organic content achieved being close to $100 \%$. These results showed the high effectiveness of the one-pot hydrothermal synthesis to introduce all the organic components used during the preparation of the layered hybrid solids.

\section{Spectroscopic characterization}

\section{NMR spectroscopy}

${ }^{13} \mathrm{C}$ CP/MAS NMR spectra of the layered organic-inorganic materials are shown in Figure 5. The spectra indicate the total integrity of the organic species located in the interlayer space after the hydrothermal synthesis processes, since all carbon atoms, including the carbon atoms directly bonded to $\mathrm{Si}$, are univocally assigned in the spectra (see the inset in Figure 5). The results confirmed that arylic and ethylendiamino bridged fragments remain intact in the as-synthesized hybrid materials (ECS-1, LHM-BTMN and LHM-TEMS) as they were in the initial bridged silsesquioxane precursors, indicating the existence of Si-bonded carbon species in the lamellar solids. It was also possible to observe, in LHM-TEMS solids, the presence of carbon atoms due to pending methyl groups covalently linked to tetrahedron silicon species from TEMS monosilanes used during the synthesis process (band centered at $-0.9 \mathrm{ppm}$ ). It is important to point out that, in all samples, the bands associated to alkoxide groups present in the starting disilanes and monosilanes were not observed, indicating that hydrolysis of all methoxy or ethoxy terminal groups of the organosilicon precursors was completed during the 
hydrothermal one-pot route. On the other hand, the ${ }^{13} \mathrm{C}$ NMR spectra of the samples obtained after the acid exchange (LHM-BTMN-H and LHM-TEMS-H) exhibited similar chemical shifts which indicate, again, that the organic fragments remained unmodified when sodium cations are replaced with protons. However, in the protonic exchanged samples, additional bands were observed due to the solvent $(\mathrm{EtOH})$ used during the consecutive washings, which remains partially occluded into the porous hybrid network after the acid post-synthesis treatments (Figure 5).

The ${ }^{13} \mathrm{C}$ NMR spectroscopy confirmed that organic fragments, such as arylic and ethylendiamino groups, preserve their integrity during the hydrothermal synthesis processes. However, ${ }^{29}$ Si MAS NMR corroborates that the organic groups not only remained intact but were also incorporated covalently into the lamellar framework, being probably bonded to tetrahedral silica units located between inorganic sheets. Figure 6 shows the ${ }^{29} \mathrm{Si} B D / M A S N M R$ spectra of different organic-inorganic layered solids prepared starting from different organosiloxanes. In all cases, characteristic bands from $-60 \mathrm{ppm}$ to $-80 \mathrm{ppm}$, assigned to T-type silicon species having a $\mathrm{Si}-\mathrm{C}$ bond, were clearly identified. Specifically, it can be seen, in the spectra containing arylic (ECS-1) and ethylendiamino (LHM-BTMN) linkers, one main broad band assigned to $\mathrm{T}$ silicon atoms $\left(\mathrm{C}-\mathrm{Si}(\mathrm{OH})_{x}(\mathrm{OAl})_{3-x}, x=0-2\right)$, being impossible to distinguish separately individual $\mathrm{T}^{1}, \mathrm{~T}^{2}$ and $\mathrm{T}^{3}$ silicon species due to the broadness of this chemical shift. These results would be indicative that the poly-condensation of organosilicon precursors may not be complete, being then possible to detect the presence of some silanol groups on the surface of the inorganic layers.

It is also important to remark the absence of Q-type silicon atoms, characteristic of tetrahedral silicon species present in highly siliceous materials, in the solids containing ethylendiamino linkers (LHM-BTMN). This confirms that cleavage of organic bridges does not occur during the one-pot alkaline hydrothermal synthesis process. On the contrary, in the described ECS-1-type materials, which contain arylic fragments in the interlayer space, an additional broad band centered at approximately $-82 \mathrm{ppm}$ is clearly observed in the ${ }^{29} \mathrm{Si}$ $\mathrm{BD} / \mathrm{MAS}$ NMR spectra, confirming that $\mathrm{Si}(n \mathrm{Al})$ sites $(n=2-4$, see Table 2$)$ are formed in ECS-1 type materials, due to the partial cleavage of the BTEB organosilicon precursor during the synthesis process $(19 \% \mathrm{~mol}){ }^{36}$ The Si-C hydrolysis was reported to occur during the preparation of hybrid phenylene-bridged materials, when the sol-gel reaction was performed under hydrothermal conditions in basic media, i. e., a methodology similar to the one followed in the present study. ${ }^{53,54,55}$ 
On the other hand, the preparation of layered hybrid materials, LHM-TEMS, by combination of organosilane and monosilane precursors resulted in hybrid materials containing standard tetrahedral silicon units $\mathrm{Si}(n \mathrm{Al})$ in their framework from TEOS. One additional chemical shift around $-85 \mathrm{ppm}$, together with T-type silicon species due to the presence of silyl-ethylendiamino and silyl-methyl groups from BTMN and TEMS precursors can be observed (Figure 6 and Table 2). Quantification of the different silicon species observed in the LHM-TEMS sample lead to $\sim 55 \%$ of silicon atoms functionalized with organic moieties instead of the $\sim 75 \%$ theoretically expected (see experimental section). This would indicate that some silicon atoms bonded to pending methyl groups suffered a cleavage process and $\sim 20 \%$ of silicon atoms from T-type are converted to $\mathrm{Si}(n \mathrm{Al})$ species. This $\mathrm{Si}-\mathrm{C}$ rupture phenomenon could be attributed only to organosilicon units provided from TEMS precursor molecules, since BTMN bridged silsesquioxanes remained intact under the synthesis conditions, as it was observed for layered hybrid materials obtained from only BTMN precursors (LHM-BTMN), in where the cleavage effect was not detected.

Additional confirmation of the incorporation of organic linkers in layered hybrid materials by binding to inorganic fragments, come from ${ }^{29} \mathrm{Si}$ NMR spectra of initial organosilicon precursors (BTEB, BTMN and TEMS). Normally, the pure organic-inorganic monomers exhibited only one peak characteristic of silicon atoms centered between $-40 \mathrm{ppm}$ and -60 ppm (T-type), depending on the organic bridging group (arylic, $\sim 59$ ppm, and ethylendiamino, $\sim-43 \mathrm{ppm}$ ) or pending (methyl) groups ( $\sim-42.5 \mathrm{ppm})$. When these organic linkers are finally bound to framework in the hybrid lamellar material, the chemical shift corresponding to silicon atoms bonded to carbon units goes from $-60 \mathrm{ppm}--80 \mathrm{ppm}$ (Table 2). This corroborates the integration of organic linkers within the final hybrid materials (see Figure 6 inset). On the other hand, similar ${ }^{29} \mathrm{Si}$ NMR spectra were obtained after strong acid treatment to exchange sodium cations, confirming the stability of the layered organicinorganic framework (Figure 6).

The presence of aluminum framework in the hybrid solids was clearly confirmed by ${ }^{27} \mathrm{Al}$ MAS/NMR chemical shifts (Figure 7). It can be observed there that the lamellar hybrid samples with arylic and ethylendiamino groups exhibited, before and after the acid exchange treatments, one main peak centered at approximately $56 \mathrm{ppm}$, which is assigned to tetrahedral aluminum. The characteristic band at $\sim 0 \mathrm{ppm}$, corresponding to octahedrally coordinated aluminum, was observed but with very reduced intensity, indicating that the majority of aluminum ( $95 \%$ ) was within the tetrahedral network of the silicoalumina layers. After the post-synthesis acid exchange treatment, the amount of NMR visible octahedral 
aluminum ( $\mathrm{Al}^{\mathrm{Vl}}$ ) increased in the layered hybrid solids (LHM-BTMN-H and LHM-TEMS-H), indicating that the acid treatment favored the formation of structural defects, together with the partial formation of amorphous species, that should be accompanied by extraction of aluminum from the silicoaluminate network. Furthermore, it was observed, in the spectra of exchanged samples (Figure $7 \mathrm{c}$ and e), a slight displacement to lower chemical shifts ( $51 \mathrm{ppm}$ ) in the band assigned to framework aluminum $\left(\mathrm{Al}^{\mathrm{IV}}\right)$, probably due to the presence of highly charged extra-framework aluminum atoms close to tetrahedrally coordinated aluminum. It would also be possible that these alterations in the chemical shifts observed from NMR spectra were due to the partial attachment of one or two water molecules on the framework aluminum species located in structural defects. ${ }^{56}$ Nevertheless, it should be noticed that after the acid exchange, $60 \% \mathrm{~mol}$ of aluminum remains tetrahedrally incorporated in the structure of the inorganic sheets. The presence of $\mathrm{Al}^{\mathrm{IV}}$ in the inorganic part of the hybrid materials after acid exchange implies the presence of bridged hydroxyls and, consequently, the existence of acid groups. So, the existence of Brönsted acid sites in the inorganic layers and base groups located in the interlayer space (LHM-BTMN-H and LHM-TEMS-H materials), from ethylendiamino groups, will result into a functional layered hybrid organic-inorganic materials with separated acid and base sites.

\section{IR spectroscopy}

Infrared (IR) spectroscopy also confirms the nature and integrity of the organic moieties present in the layered organic-inorganic hybrid materials (LHM) (Figure 8). In the case of the lamellar solid synthesized with BTMN, TEMS and TEOS as precursors (LHM-TEMS), the FTIR spectra obtained at different temperatures showed the bands associated to stretching ( $v(-$ $\left.\mathrm{NH}-): 3100-3350 \mathrm{~cm}^{-1}\right)$ and bending $\left(\delta(-\mathrm{NH}-): 1615 \mathrm{~cm}^{-1}\right)$ vibrations of secondary amines included into the propyl(ethylendiamino) bridges from BTMN silsesquioxanes. Due to the presence of these organic linkers, the $-\mathrm{CH}_{2}-$ symmetric and asymmetric vibration bands assigned to ethylen- and propylen- chains linked to amino groups $\left(\mathrm{v}\left(-\mathrm{N}-\mathrm{CH}_{2}\right): 2875\right.$ and 2930 $\mathrm{cm}^{-1} ; \delta\left(-\mathrm{N}^{-} \mathrm{CH}_{2}\right)$ : 1388 and $\left.1458 \mathrm{~cm}^{-1}\right)$ were also clearly observed. Furthermore, the characteristics stretching $\left(\mathrm{v}\left(\mathrm{Si}-\mathrm{CH}_{3}\right): 2875\right.$ and $\left.2960 \mathrm{~cm}^{-1}\right)$ and bending $\left(\delta\left(\mathrm{Si}-\mathrm{CH}_{3}\right): 1411 \mathrm{~cm}^{-1}\right)$ vibrations associated to pending methyl groups from TEMS organosilicon precursors were also detected (Figure 8a). Additional band attributed to surface silanol (Si-OH) groups, typical of inorganic silicates with structural defects, was observed at $3740 \mathrm{~cm}^{-1}$. Moreover, in the IR framework range, the characteristic band between 600 and $750 \mathrm{~cm}^{-1}$ assigned to Si-C vibration was observed, confirming the covalent and stable interaction among tetrahedron silicon units 
and organic fragments. ${ }^{57}$ These last bands were also observed together with the typical vibration bands $\left(475,865\right.$ and $\left.1005 \mathrm{~cm}^{-1}\right)$ due to the presence of T-O-T' groups which are the building units of the individual silicoaluminate sheets (see inset included in Figure 8). Following the performed thermal treatment, it is important to point out that the organic moieties, present in the materials, decompose at temperatures higher than $350^{\circ} \mathrm{C}$, as it was also observed in the thermogravimetrical analysis (Figure S3). Indeed, practically the totality of vibration bands assigned to organic fragments gradually disappeared when the temperature is increased at $350 \circ \mathrm{C}$ or above (Figure $8 \mathrm{~b}$ and $8 \mathrm{c}$ ). In conclusion, the spectroscopic results (RMN and FTIR) indicate unambiguously the existence and integrity of the organic species in the layered hybrid materials. Moreover, the spectroscopic results allow concluding that these organic linkers were covalently integrated into the framework of the solids here studied.

\section{Textural properties}

Nitrogen adsorption isotherms of the layered hybrid materials are shown in Figure 9. In all cases, the lamellar solids with arylic or ethylendiamino linkers ordered in the interlayer space, separating the silicoaluminate sheets, exhibited a conventional type II isotherms characteristic of solids with reduced measurable porosity. In fact, the hybrid materials have BET specific surface area between $60-140 \mathrm{~m}^{2} \mathrm{~g}^{-1}$ (Table 3). In these layered materials, the elevated concentration of organic linkers located in the interlayer space may not favor the existence of porous galleries with higher dimensions between the inorganic sheets, which would explain the reduced specific surface area of the layered hybrid materials here synthesized. It is also possible that delimited pores by six member rings (6MRs) were present into each individual silicoalumina layer (Figure 3$),{ }^{37,40}$ but this intracrystalline porosity is not measurable from gases adsorption techniques. So, these premises hinder the exact textural analyses of the hybrid layered samples, being complicate to estimate pore size distributions. However, textural differences were observed between ECS-1 and LHM-BTMN materials since higher surface area and porous volume were appreciated when arylic instead of ethylendiamino fragments were located in the interlayer space. This could be explained by the higher rigidity of the aromatic ring units, in BTEB, in comparison with the flexible hydrocarbonated propylamino chains, within BTMN, which could favor the formation of layered materials with a non-regular porous distribution.

With the objective to increase the pore volume and surface area of the materials, the loading of organic linkers located in the interlayer space was reduced in the synthesis by the 
partial introduction of TEMS and TEOS instead of BTMN as the only organosilicon precursor (LHM-TEMS). With this approach, a smaller amount of ethylendiamino fragments would be intercalated, and higher free porosity could be generated between the inorganic layers. The results from nitrogen adsorption isotherms (Table 3) show that, indeed, the materials with pending methyl groups' contribution onto the surface of silicoalumina sheets (LHM-TEMS) exhibited a twofold increase in surface area, in comparison with layered hybrid material prepared using only BTMN as organosilicon precursor (LHM-BTMN). Finally, it is important to remark that the strong acid exchange treatments (LHM-BTMN-H and LHM-TEMS-H) did not substantially modify the textural characteristics of the lamellar organic-inorganic hybrid materials (Figure 9).

The porous nature of the layered hybrid materials shown above, as per nitrogen adsorption measurements, would allow the calculation of potential acid sites present in the inorganic layers, due to the presence of tetrahedral aluminum, by measuring the desorbed ammonia by thermoprogrammed desorption technique (TPD). Specifically, the as-synthesized samples LHM-BTMN and LHM-TEMS desorb around $130-230 \mathrm{~cm}^{3} \mathrm{NH}^{3} \mathrm{~g}^{-1}$, increasing this amount up to $400-550 \mathrm{~cm}^{3} \mathrm{NH}_{3} \mathrm{~g}^{-1}$ in the exchanged acid samples (Table 4 and Figure S4). This result confirmed that the majority of bridged acid hydroxyl groups are compensated by sodium cations which are finally transformed in acid sites after the proton exchange process. It is important to point out the high strength of the acid sites detected in the hybrid solids because in all cases the practical totality of ammonia is desorbed between $380-480$ ㄷ (Figure S4). One additional desorption ammonia band is also observed, at approximately $200 \circ \mathrm{C}$, probably due to slight contribution of weak acid sites associated to ammonia molecules which interact with external silanol groups. Definitively, the characterization data obtained clearly corroborate the possibility to prepare well-defined layered hybrid materials with acid sites in the silicoaluminate sheets and base sites in the organic fragments located in the interlayer galleries.

\section{Catalytic activity}

The valorization of the layered hybrid materials, containing interlayered ethylendiamino linkers, as active base catalysts was considered through the condensation of carbonyl compounds with active methylenic groups which constitutes an important tool in organic chemistry for $\mathrm{C}-\mathrm{C}$ bond formation and the synthesis of substituted alkenes derivatives. These latter are of interest as end-products and intermediates in the production of fine 
chemicals and commodities (perfumes, pharmaceuticals, polymers), and we will also use the described materials for the synthesis of photosensibilizers. ${ }^{58,59,60,61,62}$

In this work, it is analyzed the catalytic activity of the more accessible LHM-TEMS-H hybrid material, after the protonic exchange, to perform condensation reactions. In this way, the presence of compensating sodium cations which could act as base catalytic centers is avoided. Firstly, the reactions between benzaldehyde and several activated methylene groups with different $p k_{a}$ substrates of 7,9 and 11, which correspond to malononitrile, ethylcyanoacetate and ethylacetoacetate, respectively (Scheme 1), were carried out to evaluate the level of basicity achieved within these materials. From the results in Figure 10, it can be observed that the condensation of benzaldehyde with malononitrile and ethylcyanoacetate was successfully performed in presence of LHM-TEMS-H material, using a 2.5 and 5 mol\% of $\mathrm{N}$, respectively. Benzylidene malononitrile and ethylbenzylidene cyanocinnamate with yield of $99 \%$ and $94 \%$ were obtained after $4.5 \mathrm{~h}$ and $3.0 \mathrm{~h}$ reaction time, respectively. Nevertheless, when the reaction was performed with ethyl acetoacetate (10 mol\% of $\mathrm{N}$ ), which requires stronger basic sites, the yield to 2-benzylidene acetoacetate was only $23 \%$, after $7 \mathrm{~h}$ of reaction time. Lower yields were also obtained with the more demanding methylenic reagent when the three considered Knoevenagel condensation tests, using different $p k_{a}$ substrates, were performed in the same reaction conditions (Figure S5). From these results, it can be concluded that ethylendiamino groups are present in the layered hybrid materials (LHM-BTMN-H) and they are accessible to reactants and active to catalyze reactions requiring relatively weak base sites.

However, the basicities achieved with this type of lamellar hybrid solids is high, enough to perform a number of reactions directed to prepare interesting chemical products. For instance, we will show below that they successfully and very selectively catalyze the synthesis of ethyl a-cyano-4-hydroxy-3-methoxycinnamate and 2-cyano-3-(4-hydroxy-3,5dimethoxyphenyl) 2-propenoic acid ethyl ester by Knoevenagel condensation between ethylcyanoacetate and vanillin, and ethylcyanoacetate and syringaldehyde (Scheme 2). The two compounds are photostabilizers used in the formulation of sunscreens. ${ }^{63,64}$ They exhibits sunscreen activity, are chromophoric within ultraviolet radiation and present antioxidant properties. The catalytic results showed that it was possible to produce ethyl $\alpha$-cyano-4hydroxy-3-methoxycinnamate with $97 \%$ of yield after $5 \mathrm{~h}$ of reaction time (Figure 11) and 2cyano-3-(4-hydroxy-3,5-dimethoxyphenyl) 2-propenoic acid ethyl ester (ethyl syringyl cyanoacetate, 4 ) with $99 \%$ of yield, after $13 \mathrm{~h}$ of reaction time (Figure 12), using 3.4 and 2.5 mol\% of LHM-TEMS-H catalyst, respectively. 


\section{Catalyst recyclability}

The recyclability of LHM-TEMS-H was examined in the Knoevenagel condensation of benzaldehyde and ethylcyanoacetate for 6 successive uses (Figure S6). After each experiment, the catalyst was filtered with acetone, dried in air and reused. We observed that the catalyst was successfully reused for 4 consecutive runs. In the fifth use, we observed the partial deactivation of the catalyst which could be regenerated by three successive thermal treatments at $60 \stackrel{\circ}{ }$ in aqueous ammonia for $2 \mathrm{~h}$. Then, in the sixth use, the catalytic properties of the material were recovered. The elemental analysis of the used LHM-TEMS-H confirmed the stability of organic functional fragment (BTMN) since nitrogen content was maintained. Thus, the catalytic results clearly evidenced the capacity of these layered hybrid materials to be used as effective and reusable base catalysts, taking advantage of the reactivity of the heterogeneized and stabilized active centers located between silicoaluminate layers.

Different catalytic tests were also carried out to evaluate the reactivity of Brönsted acid sites present in the inorganic layers due to the presence of framework aluminum. Specifically, we checked the acid catalytic properties of LHM-TEMS-H material for 3,3 dimethylbutene isomerization (at $200^{\circ} \mathrm{C}$, in fixed bed), acetal formation (dimethyl acetal of valeraldehyde, 1, 2-propanediol acetal of valeraldehyde) or acetal hydrolysis (dimethyl benzaldehyde acetal). ${ }^{65}$ Nevertheless, the solid catalyst remained inactive in the different studied processes. In this case, the high concentration of stabilized acid centers in the lamellar framework (responsible of very weak acid strength of aluminum centers), the hydrophilic behavior of the catalyst surface due to silanol groups combined with high Si/Al ratio, and the diffusional restriction of the structure can be responsible for the lack of acid catalytic activity of these materials. ${ }^{66}$ Current work is directed into the decrease of the Si/Al ratio within the inorganic component of the hybrid material.

\section{Conclusions}

Novel type of layered hybrid materials (LHMs), based on ordered aluminosilicate sheets and organic intercalated pillars (arylic and ethylendiamino units), were prepared by direct alkaline hydrothermal synthesis without previous swelling and/or pillarization processes of starting layered inorganic precursors, easing the synthesis of expanded and functional organic-inorganic lamellar materials. The direct intercalation of ethylendiamino groups from bridged silsesquioxane monomers, located in the interlayer space, together with the effective exchange of intracrystalline sodium cations by proton species, without modify the lamellar 
nature of the solids, was decisive to generate hybrid materials with located acid and base centers. Optimization of the synthesis method was studied, being possible to increase the accessibility of active sites through the reduction in the concentration of organic fragments directly intercalated between the individual aluminosilicate layers. The activity of the layered hybrid materials was verified through their use and recyclability as base catalysts for C-C bond forming reactions (Knoevenagel condensations). The obtained results would imply an important advance in the development of bi-functional hybrid materials based on ordered inorganic silicoaluminate layers (ECS-type) and organic intercalated pillars, synthesized by direct synthesis, which exhibit high catalytic reactivity.

\section{Acknowledgments}

The authors thank financial support to Spanish Government by Consolider-Ingenio MULTICAT CSD2009-00050, MAT2011-29020-C02-01 and Severo Ochoa Excellence Program SEV-2012-0267. AG and JMM thank pre-doctoral fellowships from MINECO for economical support.

Electronic supplementary information (ESI) available: schematic representation of layered hybrid materials (S1), representation of ECS materials (S2), thermogravimetical curves (TGA) and their derivatives (DTA) of LHM samples (S3), $\mathrm{NH}_{3}$ thermoprogrammed desorption curves of LHM samples (S4), catalytic performances from Knoevenagel condensations (S5), catalytic recycles (S6) and procedure to evaluate interlayered ethylendiamino groups through acid-base titration. 


\section{Tables}

Table 1. Chemical analysis of layered hybrid materials.

\begin{tabular}{|c|c|c|c|c|c|c|c|c|c|c|c|c|}
\hline \multirow[t]{2}{*}{ Samples } & \multirow[t]{2}{*}{$\%$ Silane ${ }^{a}$} & \multirow[t]{2}{*}{$c^{b}$} & \multirow[t]{2}{*}{$\mathbf{N}^{b}$} & \multirow[t]{2}{*}{$C / N^{c}$} & \multicolumn{2}{|c|}{ Org.Cont ${ }^{\mathrm{b}}$. } & \multirow[t]{2}{*}{$\mathrm{Na}^{\mathrm{b}}$} & \multirow[t]{2}{*}{$\mathrm{Si}^{\mathrm{b}}$} & \multirow[t]{2}{*}{$A I^{b}$} & \multirow{2}{*}{$\mathrm{Si} / \mathrm{Al}^{\mathrm{c}}$} & \multirow[t]{2}{*}{$\mathrm{Na} / \mathrm{Si}^{\mathrm{c}}$} & \multirow[t]{2}{*}{$\mathrm{Na} / \mathrm{Al}^{\mathrm{c}}$} \\
\hline & & & & & $\mathrm{CHN}^{\mathrm{d}}$ & TGA $^{\mathrm{e}}$ & & & & & & \\
\hline $\begin{array}{l}\text { LHM- } \\
\text { BTMN }\end{array}$ & $100 \%$ & 21.9 & 5.4 & 4.7 & 32.5 & 33.1 & 4.1 & 13.8 & 11.3 & 1.2 & 0.4 & 0.4 \\
\hline $\begin{array}{c}\text { LHM- } \\
\text { BTMN-H }\end{array}$ & BTMN & 25.6 & 6.9 & 4.3 & 38.8 & 40.4 & 0.0 & 14.8 & 11.4 & 1.2 & 0.0 & 0.0 \\
\hline $\begin{array}{l}\text { LHM- } \\
\text { TEMS }\end{array}$ & $\begin{array}{c}50 \% \\
\text { BTMN } \\
25 \%\end{array}$ & 12.9 & 2.9 & 5.2 & 19.7 & 21.1 & 9.1 & 12.2 & 15.1 & 0.8 & 0.9 & 0.7 \\
\hline $\begin{array}{l}\text { LHM- } \\
\text { TEMS-H }\end{array}$ & $\begin{array}{c}\text { TEMS } \\
25 \% \\
\text { TEOS } \\
\end{array}$ & 15.8 & 3.8 & 4.9 & 24.5 & 27.2 & 0.0 & 17.1 & 14.3 & 1.1 & 0.0 & 0.0 \\
\hline ECS-1 & $\begin{array}{l}100 \% \\
\text { BTEB }\end{array}$ & 14.7 & 0.0 & - & 17.6 & 19.8 & 7.1 & 15.6 & 10.4 & 1.4 & 0.6 & 0.8 \\
\hline
\end{tabular}


Table 2. Silicon chemical shifts for $\mathrm{T}$ and Si(nAl) species (in ppm) from ${ }^{29} \mathrm{Si} B D / M A S$ NMR spectra of layered hybrid materials and pure organosilicon precursors. In parenthesis are indicated the percentage of each silicon specie band compared with the total silicon moles.

\begin{tabular}{|c|c|c|c|c|}
\hline Samples & $\%$ Silane ${ }^{a}$ & $\mathbf{T}$ & $\mathrm{C}-\mathrm{Si}^{\mathbf{b}}$ & $\operatorname{Si}(n A I)$ \\
\hline $\begin{array}{c}\text { LHM-BTMN } \\
\text { LHM-BTMN-H }\end{array}$ & $100 \%$ BTMN & $\begin{array}{l}-56.3(100) \\
-58.1(100)\end{array}$ & -42.9 & $\begin{array}{l}- \\
-\end{array}$ \\
\hline $\begin{array}{c}\text { LHM-TEMS } \\
\text { LHM-TEMS-H }\end{array}$ & $\begin{array}{l}50 \% \text { BTMN } \\
25 \% \text { TEMS } \\
25 \% \text { TEOS }\end{array}$ & $\begin{array}{l}-57.2(57) \\
-56.5(56)\end{array}$ & $\begin{array}{l}-42.9 \text { (BTMN) } \\
-42.4 \text { (TEMS) }\end{array}$ & $\begin{array}{l}-86.8(43) \\
-86.9(44)\end{array}$ \\
\hline ECS-1 & 100\% BTEB & $\begin{array}{l}-68.9\left(T^{2}: 46\right) \\
-71.1\left(T^{3}: 35\right)\end{array}$ & -59.3 & $-81.9(19)$ \\
\hline
\end{tabular}

\footnotetext{
${ }^{a}$ Moles percentage of silanes employed during the synthesis respect total $\mathrm{SiO}_{2}$ moles. ${ }^{b}$ Chemical shift of pure organosilicon (BTEB, BTMN and TEMS) precursors.
} 
Table 3. Surface area and pore volume of layered hybrid materials estimated from adsorption nitrogen isotherms.

\begin{tabular}{|c|c|c|c|}
\hline Samples & $\%$ Silane ${ }^{a}$ & $\begin{array}{c}\mathrm{S}_{\mathrm{BET}} \\
\left(\mathrm{m}^{2} \mathrm{~g}^{-1}\right)\end{array}$ & $\begin{array}{c}V_{\text {Total }} \\
\left(\mathrm{cm}^{3} \mathrm{~g}^{-1}\right)\end{array}$ \\
\hline LHM-BTMN & & 58.9 & 0.50 \\
\hline LHM-BTMN-H & $100 \%$ BTMN & 58.7 & 0.56 \\
\hline LHM-TEMS & $50 \%$ BTMN & 135.5 & 0.47 \\
\hline LHM-TEMS-H & $\begin{array}{l}25 \% \text { TEMS } \\
25 \% \text { TEOS }\end{array}$ & 104.5 & 0.52 \\
\hline ECS-1 & 100\% BTEB & 99.6 & 0.70 \\
\hline
\end{tabular}


Table 4. Ammonia thermoprogrammed desorption of the layered hybrid materials.

\begin{tabular}{cccc}
\hline Samples & \%Silane ${ }^{\mathbf{a}}$ & $\begin{array}{c}\mathbf{N H}_{\mathbf{3}} \text { Quantity } \\
\left(\mathbf{c m}^{\mathbf{3}} \mathbf{g}^{-\mathbf{1}}\right)\end{array}$ & $\begin{array}{c}\text { Temperature at } \\
\text { Maximum (C) }\end{array}$ \\
\hline LHM-BTMN & \multirow{2}{*}{$100 \%$ BTMN } & 126.5 & 486.8 \\
LHM-BTMN-H & 559.6 & 379.6 \\
\hline LHM-TEMS & $50 \%$ BTMN & 237.4 & 395.4 \\
LHM-TEMS-H & 25\% TEMS & 407.6 & 368.5 \\
\hline
\end{tabular}

aoles percentage of silanes employed during the synthesis respect total $\mathrm{SiO}_{2}$ moles. 


\section{Schemes}

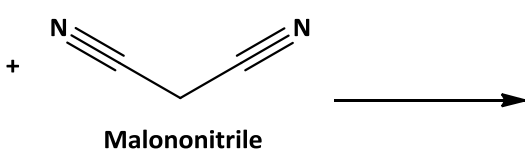<smiles>O=Cc1ccccc1</smiles>

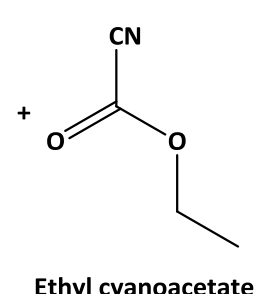

Ethyl cyanoacetate

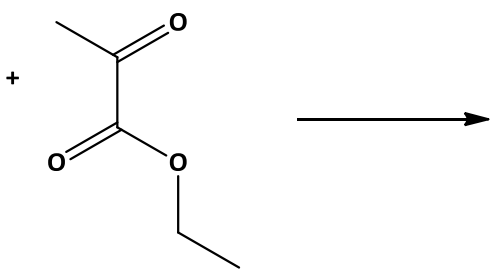

Ethyl acetoacetate

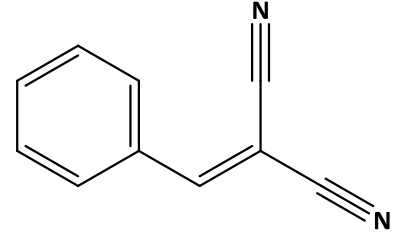

Benzylidene malononitrile<smiles>CCOC(=O)C(C#N)=Cc1ccccc1</smiles>

Ethyl benzylidene cyanocinnamate<smiles>CCOC(=O)C(=Cc1ccccc1)C(C)=O</smiles>

Ethyl 2-benzylidene acetoacetate

Scheme 1. Knoevenagel condensation between benzaldehyde and different base-strength demanding substrates. 

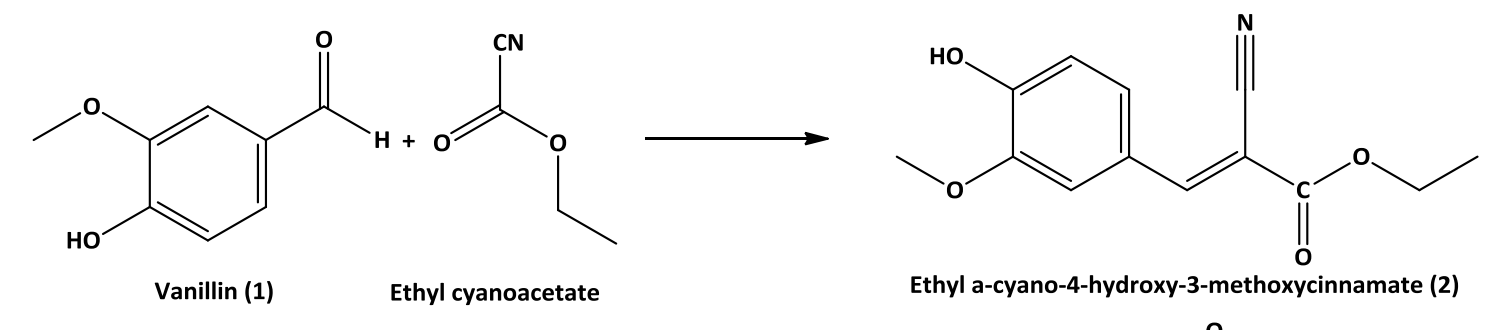<smiles>COc1cc(C=O)cc(OC)c1O</smiles>

Syringaldehyde (3)<smiles>CCOC(=O)C#N</smiles>

Ethyl cyanoacetate<smiles>CCOC(=O)C(C#N)=Cc1cc(O)c(O)c(OC)c1</smiles>

2-cyano-3-(4-hydroxy-3,5-dimethoxyphenyl) 2-propenoic acid ethyl ester (4)

Scheme 2. Knoevenagel condensation between ethylcyanoacetate and vanillin or syringaldehyde to produce 2-cyano-4-hydroxy-3-methoxycinnamate and 2-cyano-3-(4-hydroxy-3,5-dimethoxyphenyl) 2propenoic acid ethyl ester. 


\section{Figures}

(a)

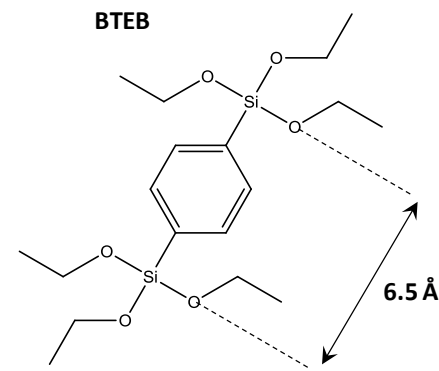

BTMN

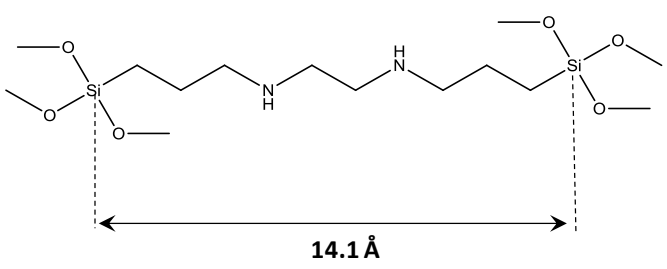

(b)

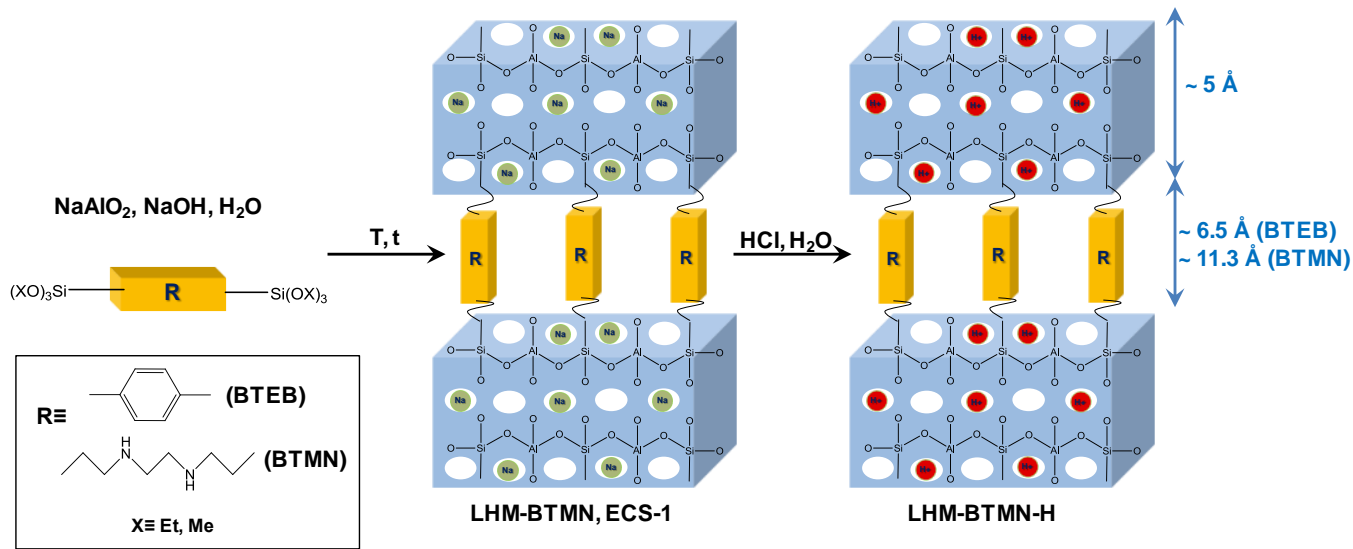

Na $\mathrm{H}_{+}$

(c)

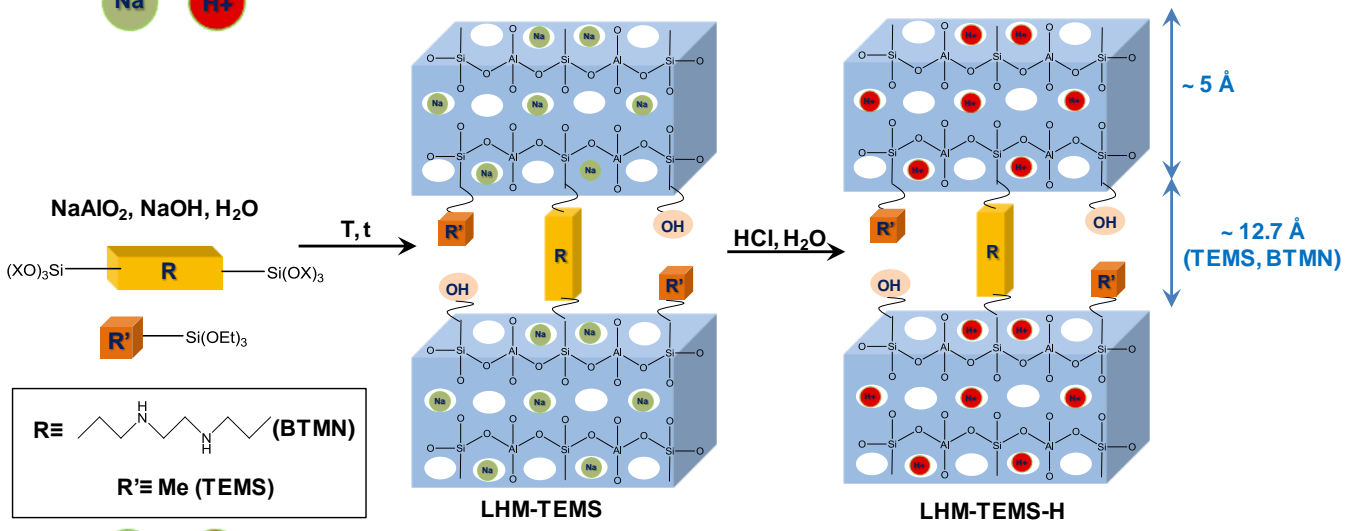

$\mathrm{Na}$

Figure 1. (a) Bridged silsesquioxanes used as organosilica precursors (BTEB: Bis(triethoxysilyl)benzene, BTMN: Bis(trimethoxysilylpropyl)ethylendiamino), (b) and (c) synthesis route followed to obtain the layered hybrid materials (LHM). 


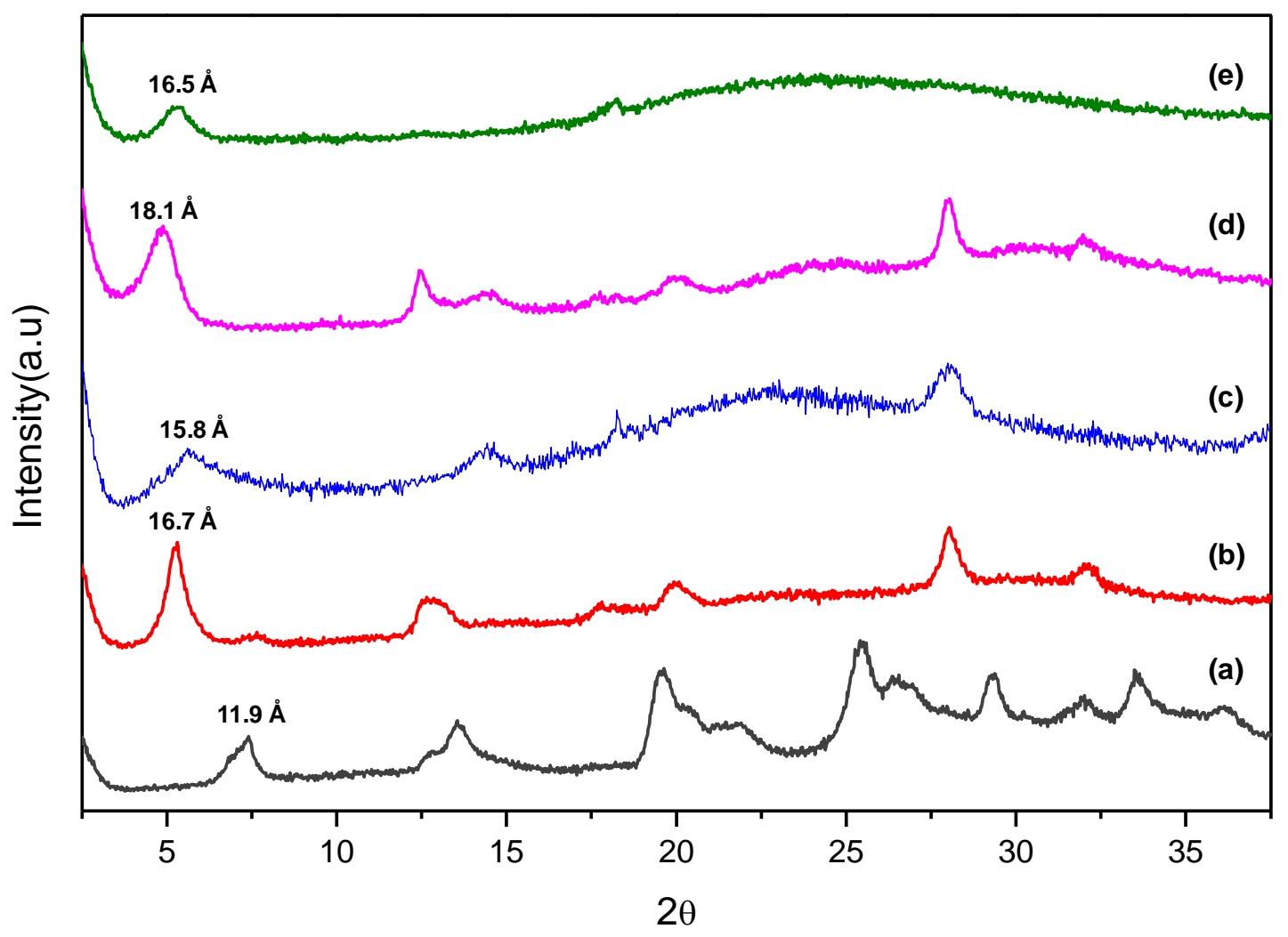

Figure 2. X-ray difractograms (XRD) of hybrid layered materials: (a) ECS-1, (b) LHM-BTMN, (c) LHMBTMN-H, (d) LHM-TEMS and (e) LHM-TEMS-H. 


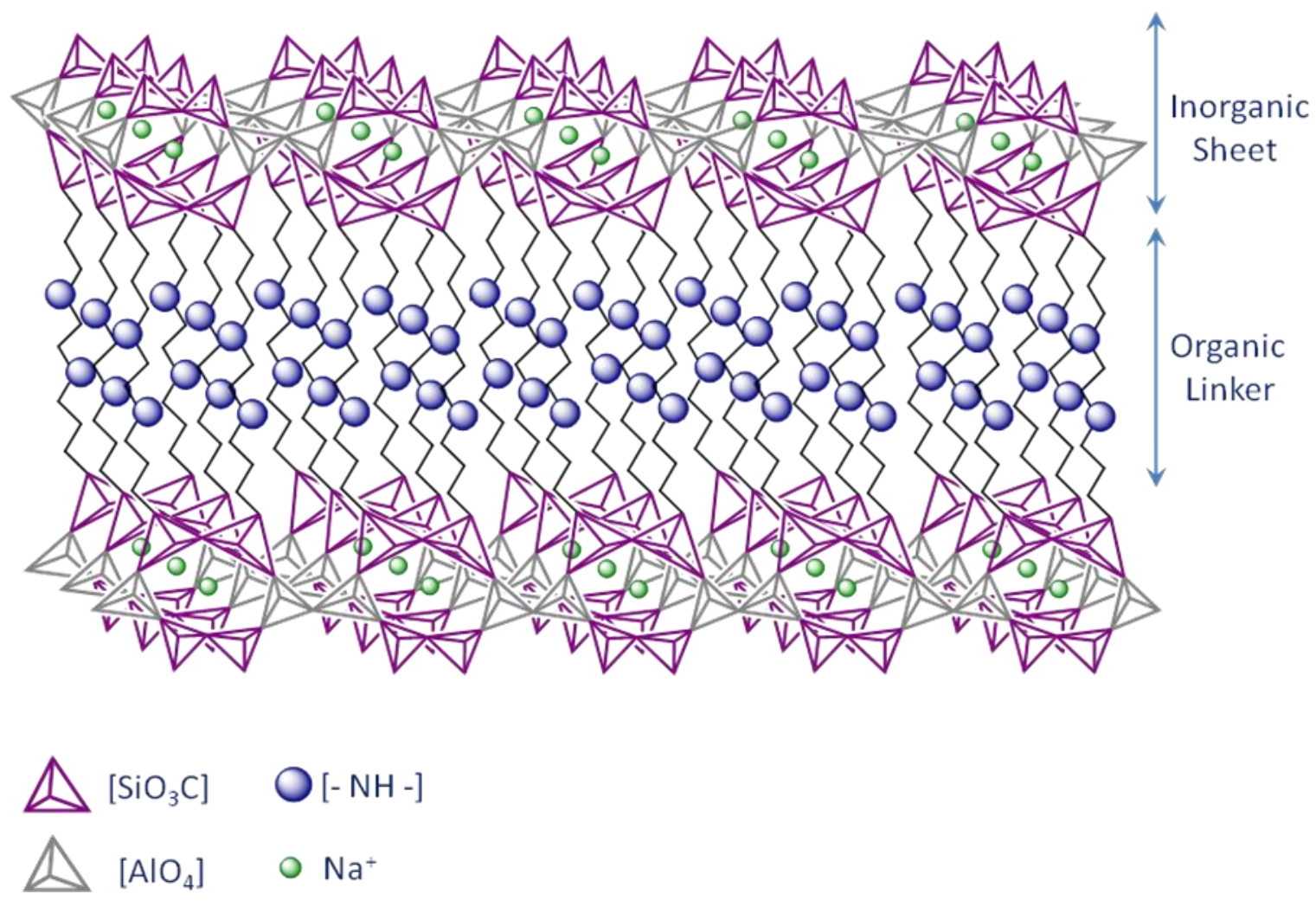

Figure 3. Artistic representation of layered hybrid materials LHM-BTMN with ethylendiamino groups located in the interlayer space between aluminosilicate layers. 

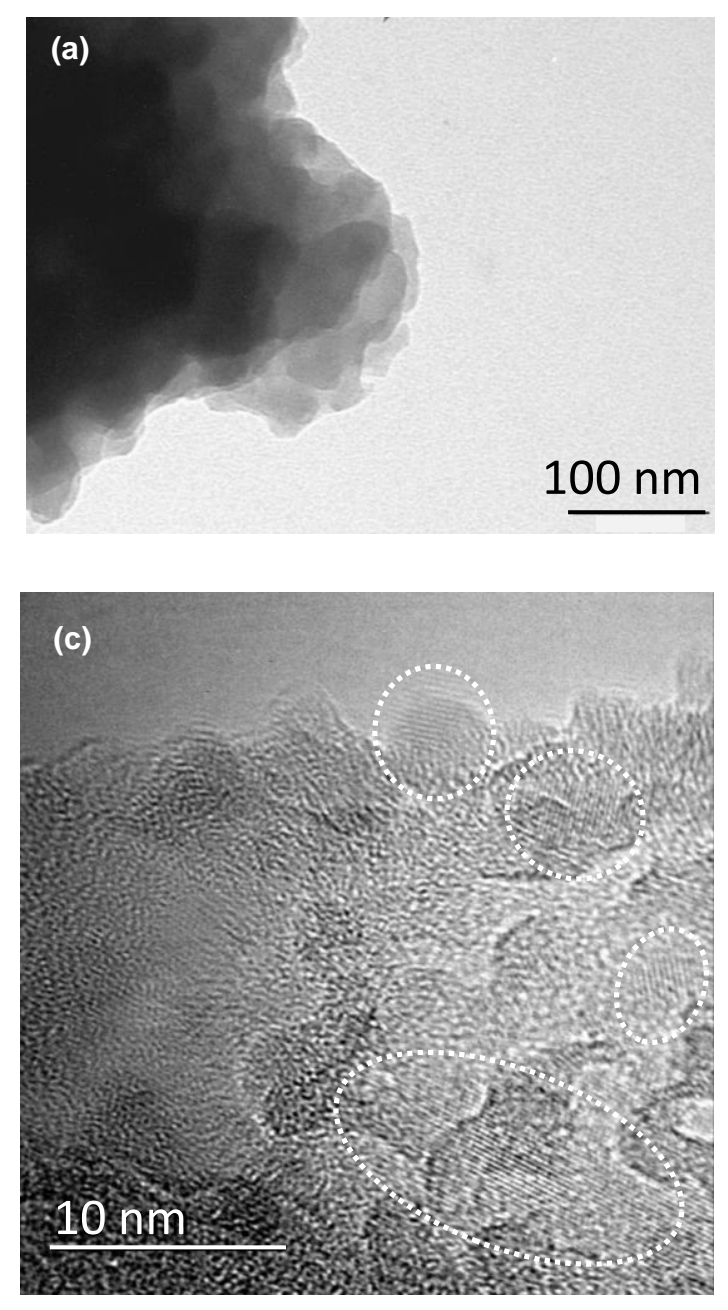
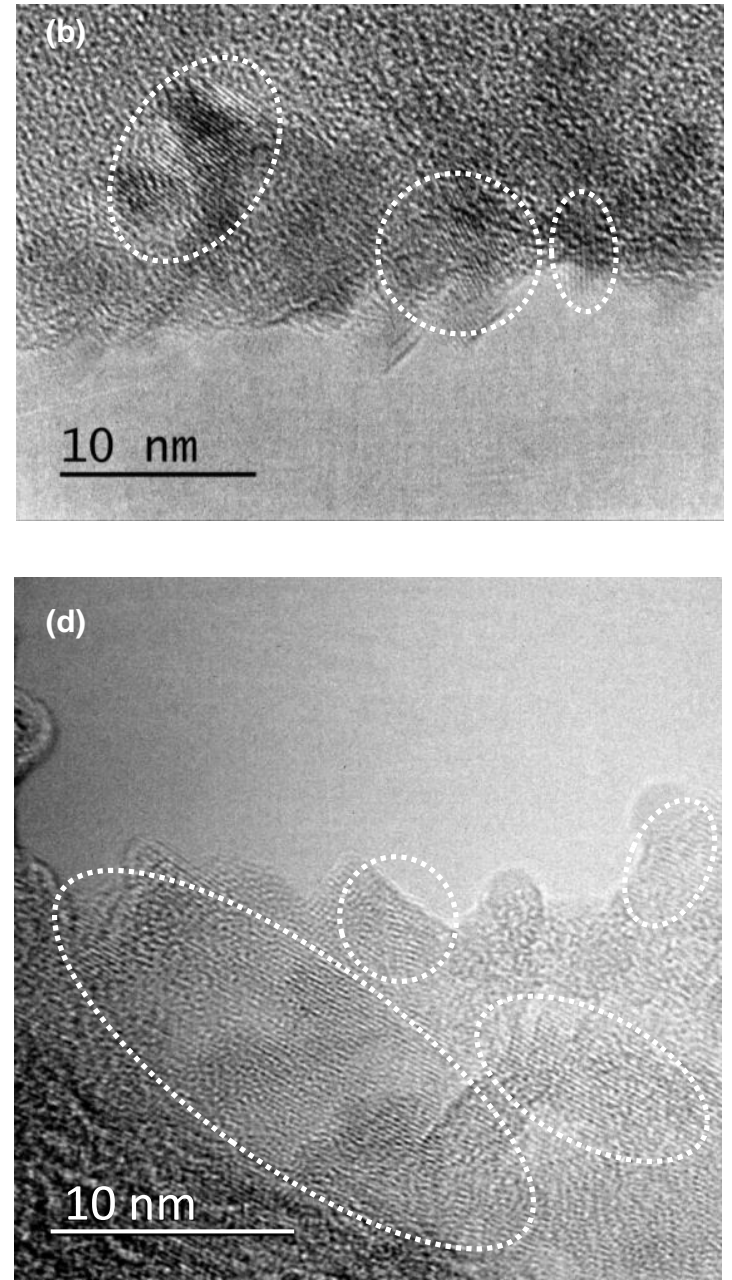

Figure 4. TEM micrographs of layered hybrid materials: (a) LHM-BTMN, (b), (c) and (d) LHM-TEMS. Inserted dashed circles highlighted lamellar organization. The reference bars correspond to $100 \mathrm{~nm}$ for (a) and $10 \mathrm{~nm}$ for (b), (c) and (d) micrographs. 


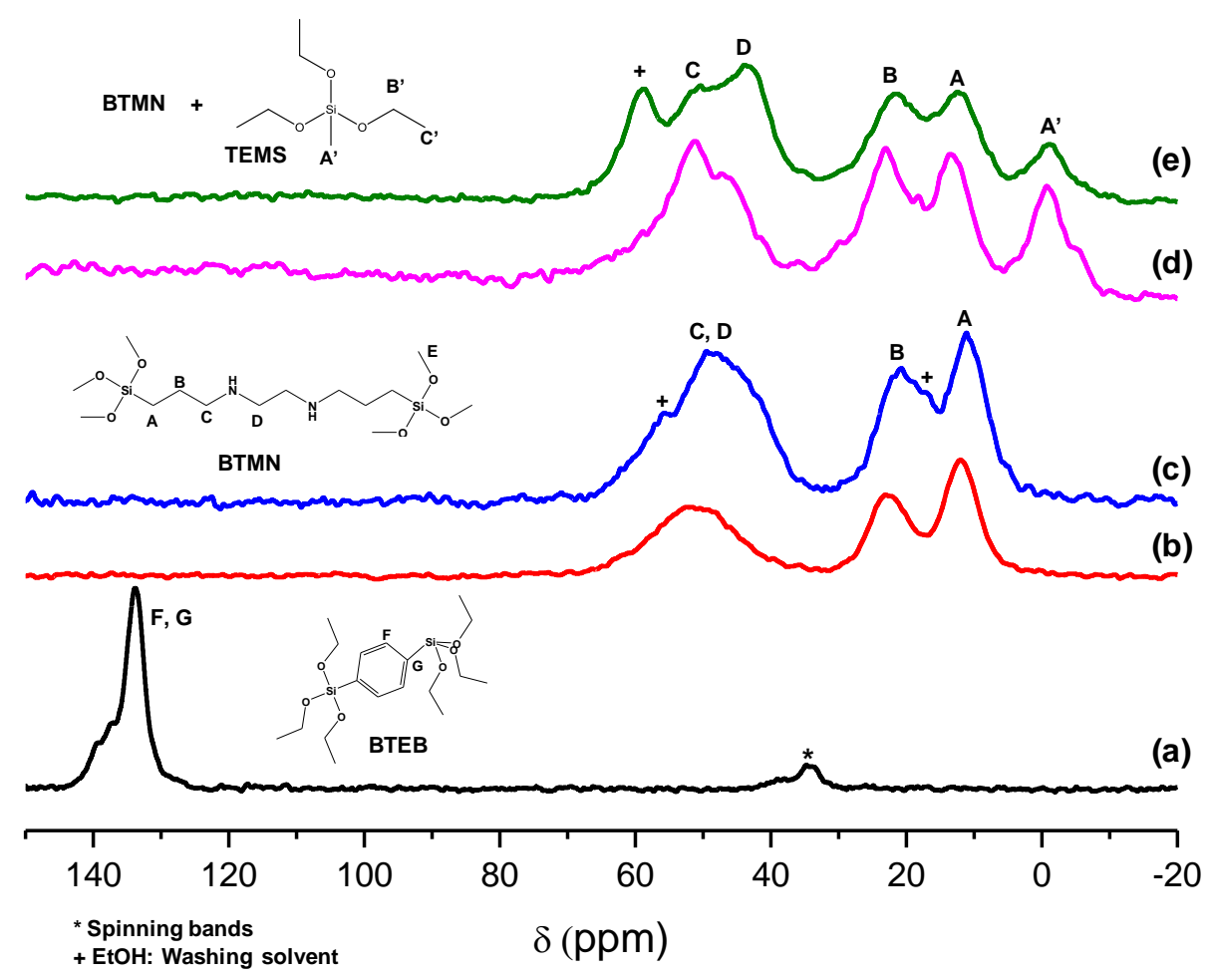

Figure 5. ${ }^{13} \mathrm{C} C P / M A S$ NMR spectra of the hybrid layered samples: (a) ECS-1-type, (b) LHM-BTMN, (c) LHM-BTMN-H, (d) LHM-TEMS and (e) LHM-TEMS-H. 


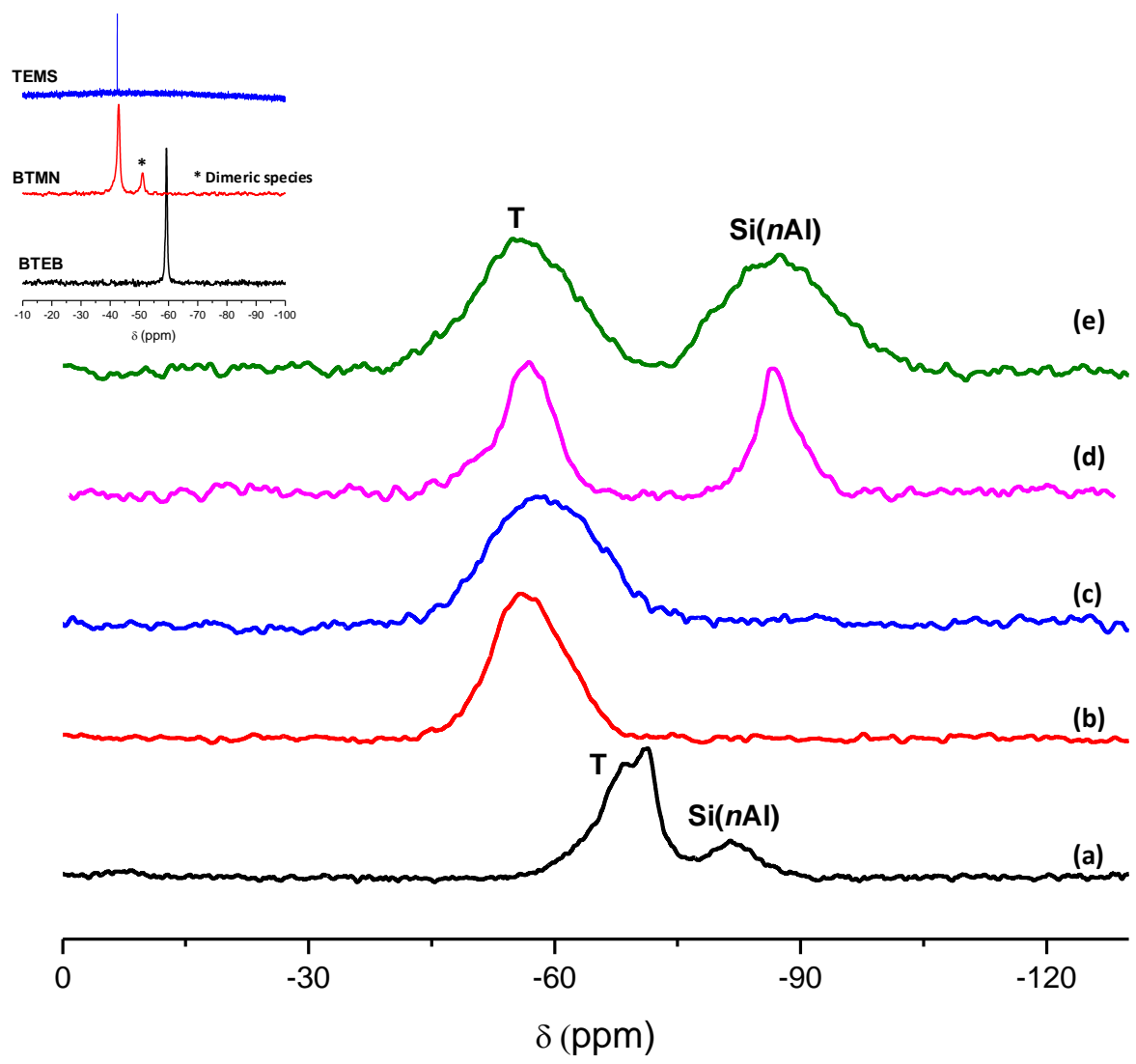

Figure 6. ${ }^{29} \mathrm{Si} \mathrm{BD} / \mathrm{MAS}$ NMR spectra of the hybrid layered samples: (a) ECS-1-type, (b) LHM-BTMN, (c) LHM-BTMN-H, (d) LHM-TEMS and (e) LHM-TEMS-H. In the inset, ${ }^{29} \mathrm{Si}$ BD/MAS NMR spectra of pure silsesquioxane precursors are shown. 


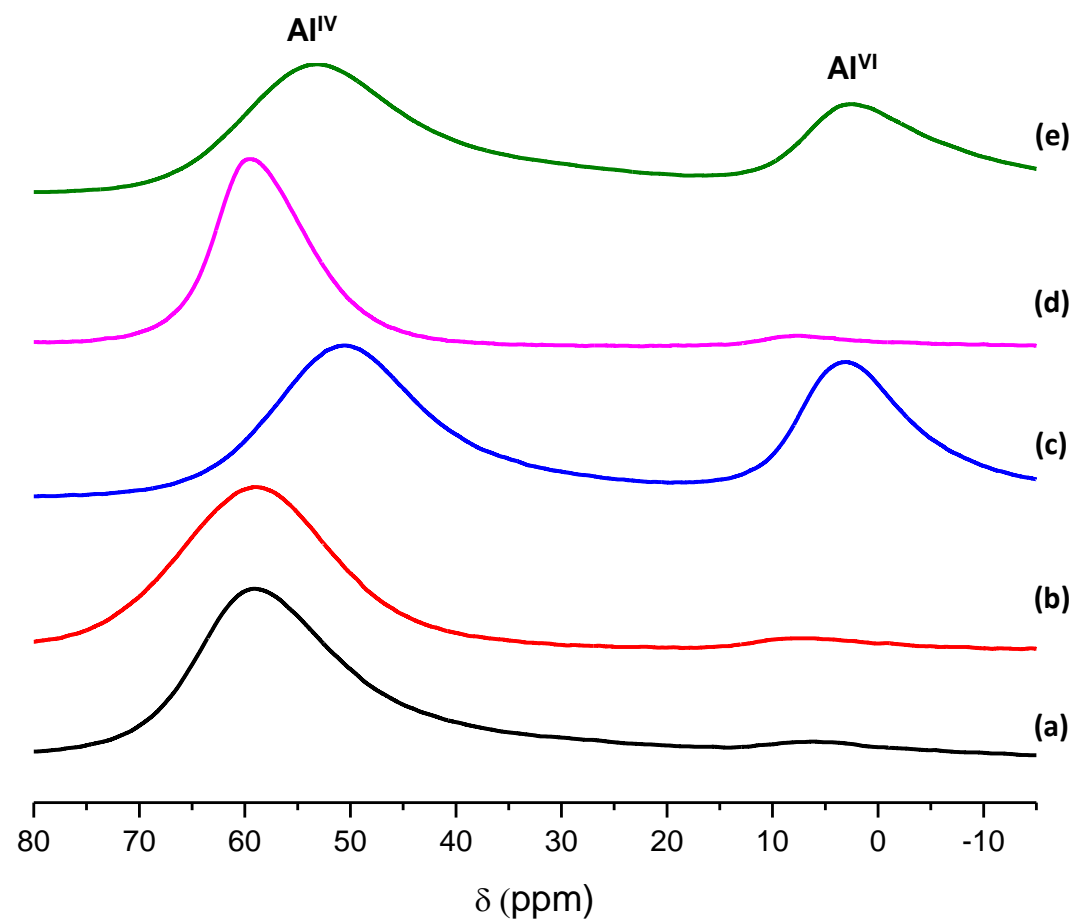

Figure 7. ${ }^{27} \mathrm{Al} \mathrm{BD} / \mathrm{MAS}$ NMR spectra of the hybrid layered samples: (a) ECS-1-type, (b) LHM-BTMN, (c) LHM-BTMN-H, (d) LHM-TEMS and (e) LHM-TEMS-H. 


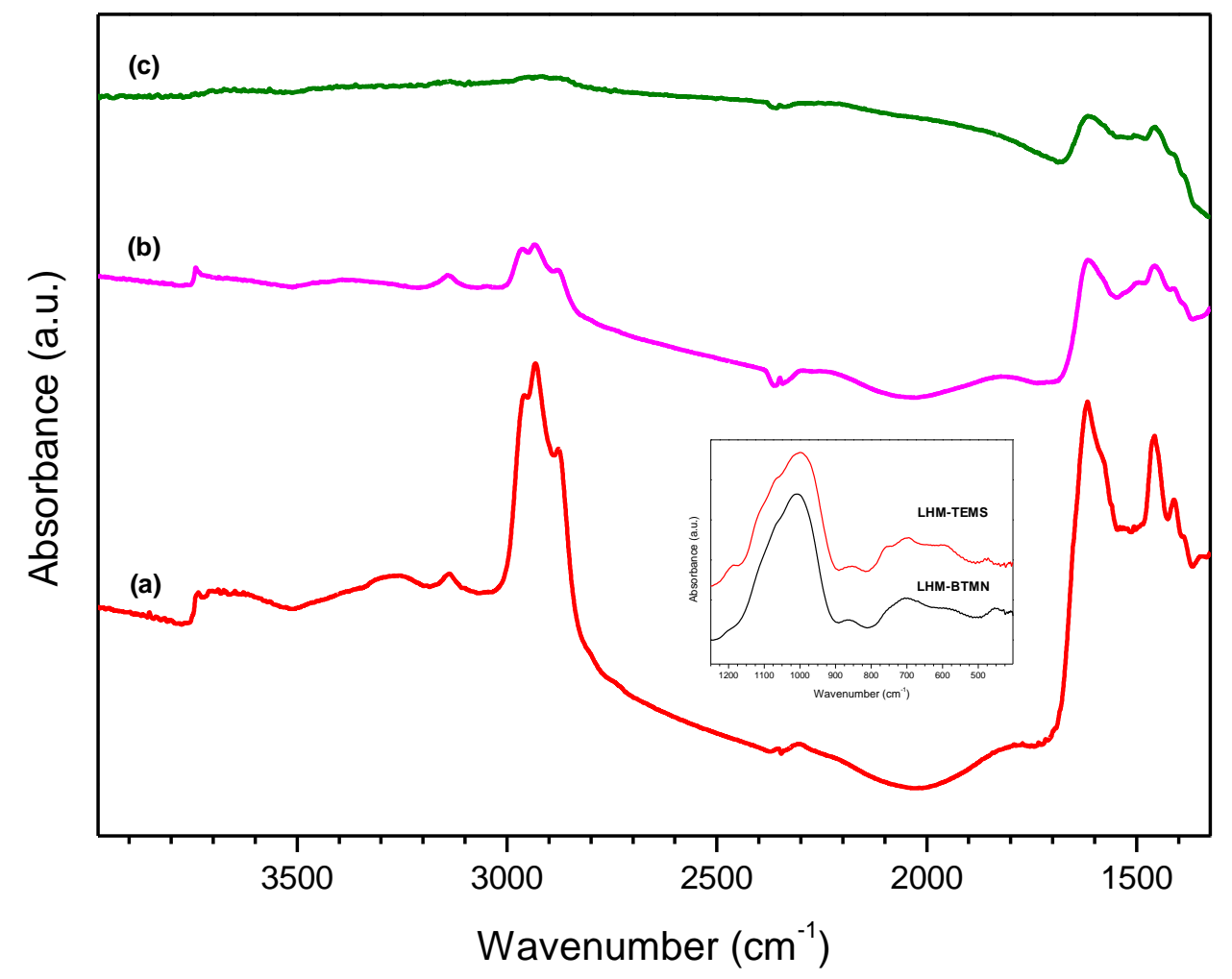

Figure 8. FTIR spectra of LHM-TEMS material at different temperatures: (a) $200 \circ \mathrm{C}$, (b) $300 \circ \mathrm{C}$ and (c) 350 ․ . In the inset, it is shown the FTIR spectra of LHM-TEMS and LHM-BTMN samples in the framework range. 


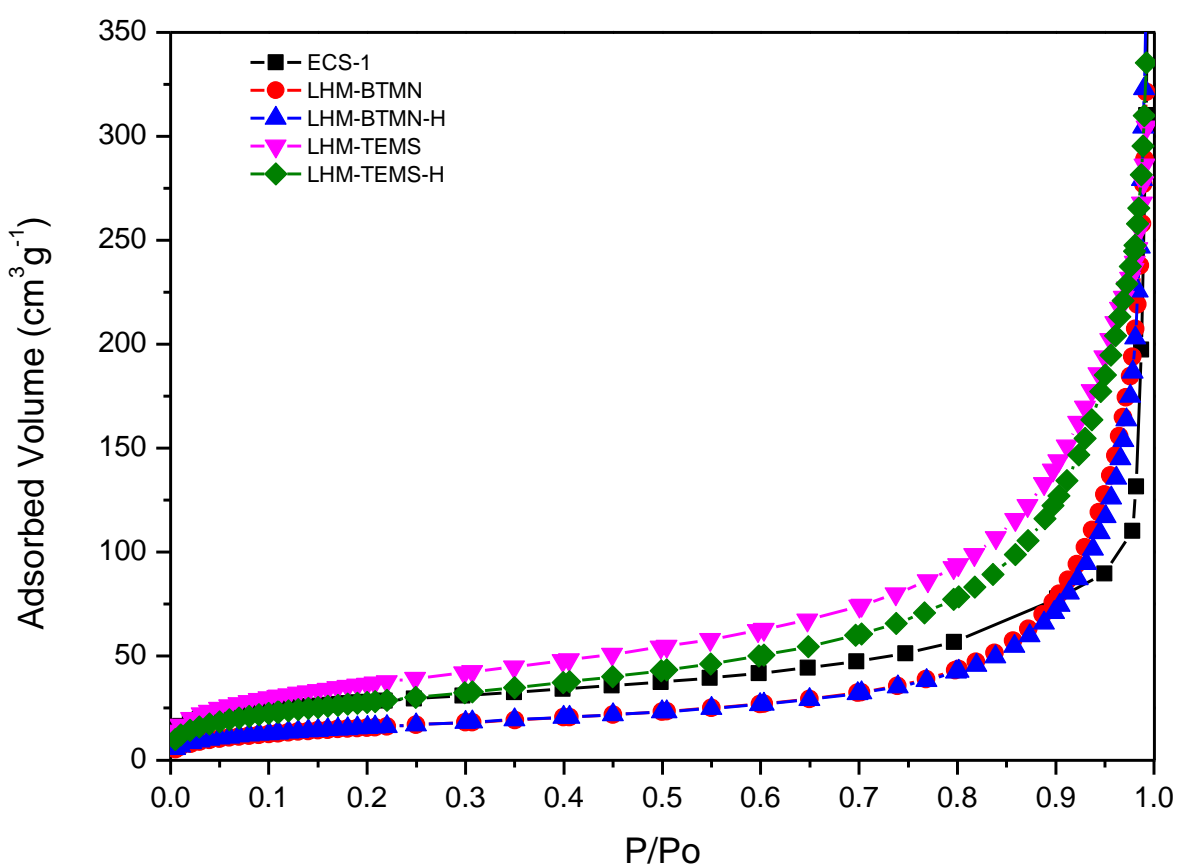

Figure 9. $\mathrm{N}_{2}$ adsorption isotherms of layered hybrid materials. 


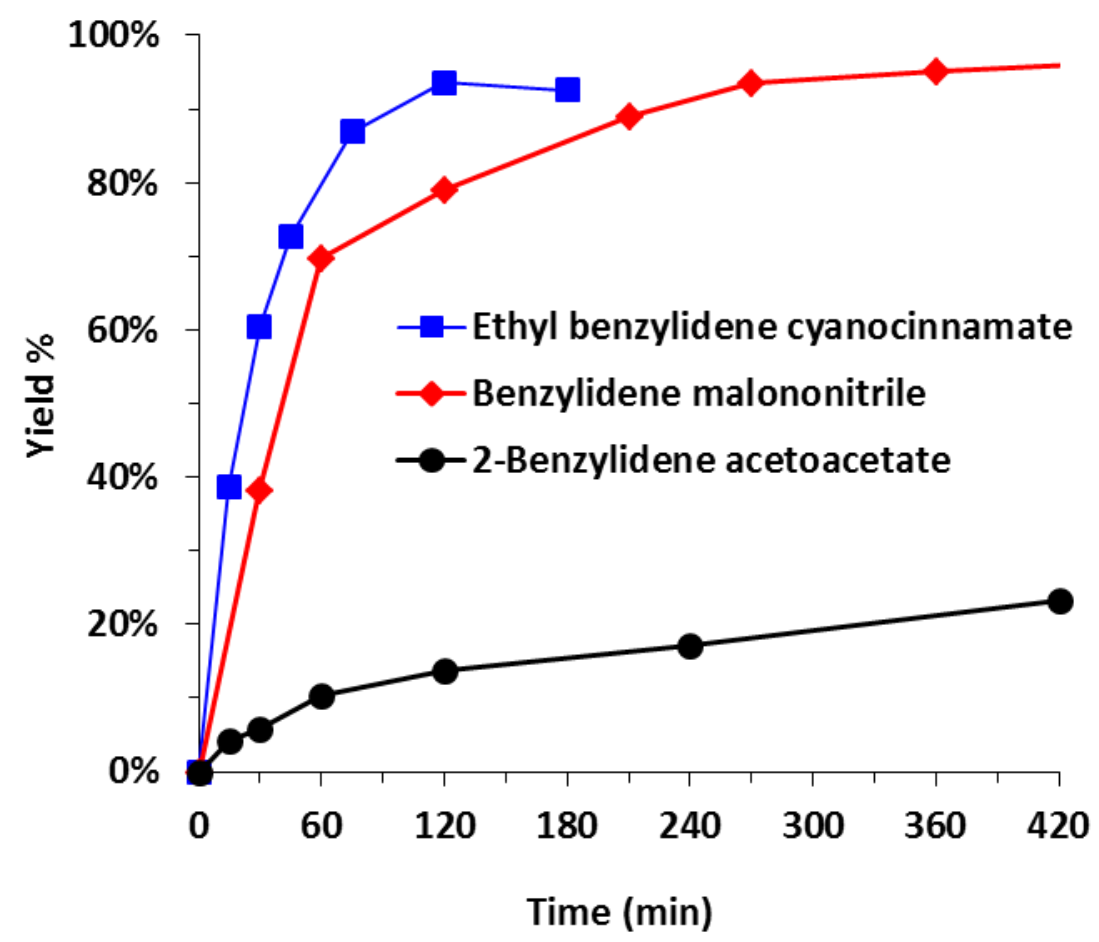

Figure 10. Benzylidene malononitrile $(\boldsymbol{\nabla})$, ethyl benzylidene cyanocinnamate ( $\boldsymbol{\square})$ and ethyl 2benzylidene acetoacetate $(\bullet)$ yields versus time when the Knoevenagel reactions were carried out in presence of LHM-TEMS-H material. 


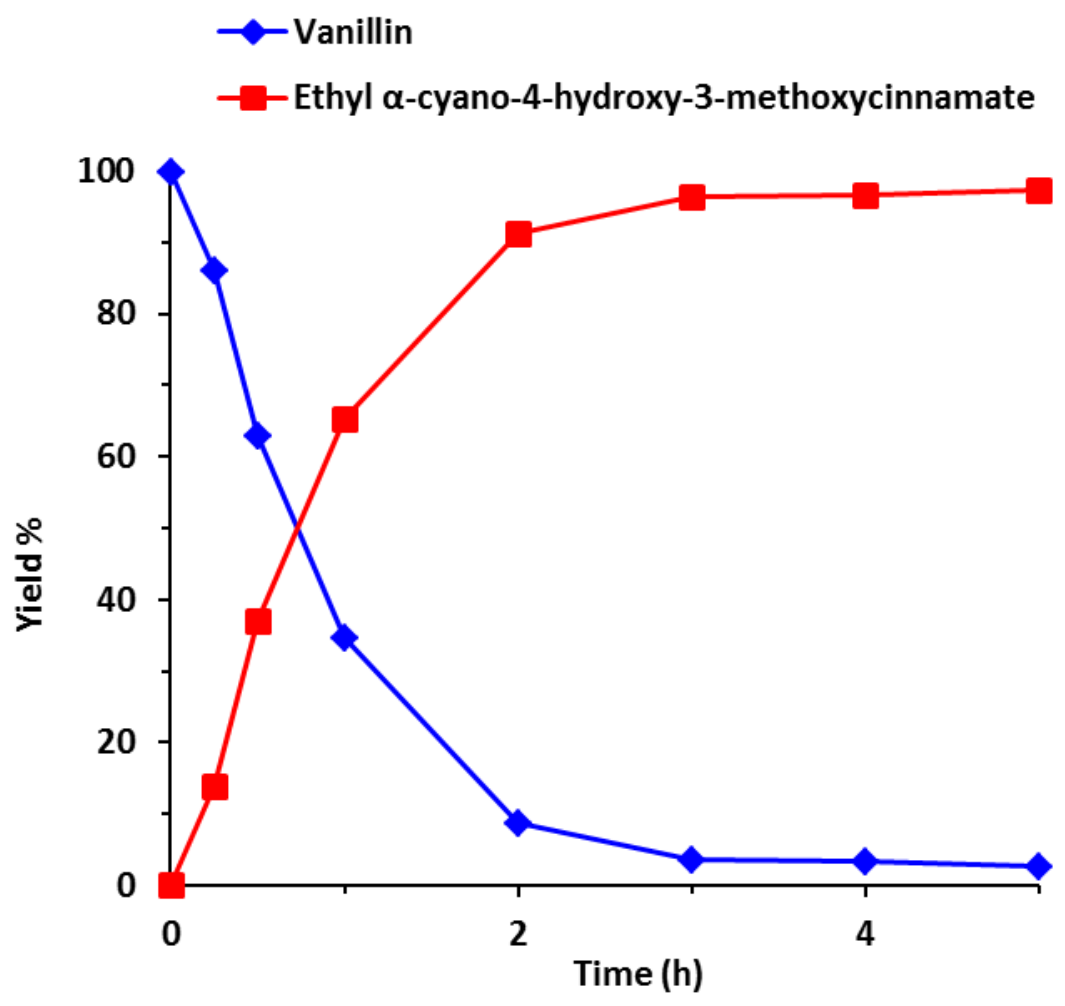

Figure 11. Ethyl syringyl cyanoacetate and syringaldehyde yields versus time when the reaction was carried out in presence of LHM-TEMS-H material as catalyst at 70 o C, with 3.4 mol\% of active sites in acetonitrile as solvent. 


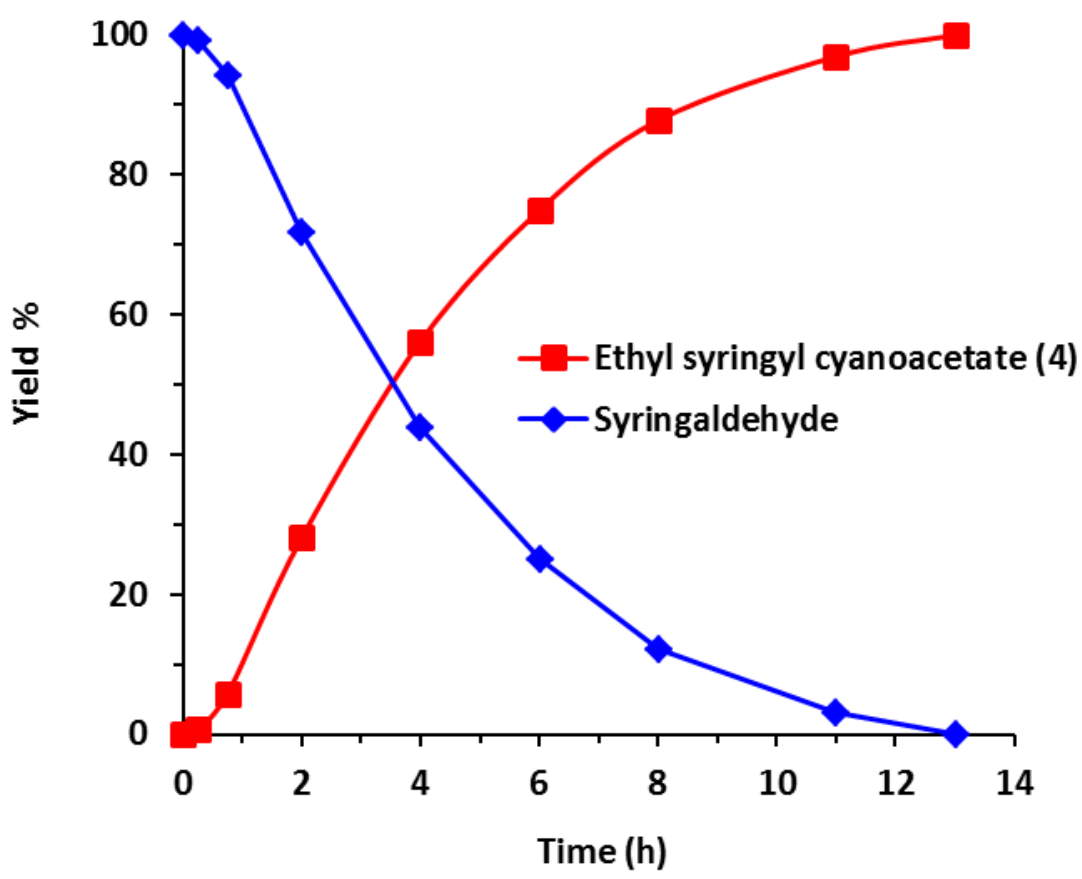

Figure 12. Ethyl $\alpha$-cyano-4-hydroxy-3-methoxycinnamate and vanillin yields versus time when the reaction was carried out in presence of LHM-TEMS-H material as catalyst at $70 \circ \mathrm{C}$, with $2.5 \mathrm{~mol} \%$ of active sites in dimethylformamide as solvent. 


\section{References}

1 (a) T. Fontecave, C. Sánchez, T. Azais and C. Boissiere, Chem. Mater., 2012, 24, 4326-4336.; (b) G.L. Drisko and C. Sánchez, Eur. J. Inorg. Chem., 2012, 32, 5097-5105; (c) L. Nicole, C. Laberty-Robert, L. Rozes and C. Sánchez, Nanoscale, 2014, 6, 6267-6292.

2 (a) A.P. Wight and M.E. Davis, Chem. Rev., 2002, 102, 3589-3614; (b) G. Férey, Chem. Soc. Rev., 2008, 37, 191-214; (c) F. Hoffmann, M. Cornelius, J. Morell and M. Fröba, Angew. Chem. Int. Ed., 2006, 45, 3216-3251.

3 C. Sánchez, C. Boissiere, S. Cassaignon, C. Chaneac, O. Durupthy, M. Faustini, D. Grosso, C. LabertyRobert, L. Nicole, D. Portehault et al., Chem. Mater., 2014, 26, 221-238.

4 C. Sánchez, B. Julián, P. Belleville and M. Popall, J. Mater. Chem., 2005, 15, 3559-3592.

5 C. Sánchez and F. Ribot, New J. Chem., 1994, 18, 1007-1047.

6 D.A. Loy and J. Shea, Chem. Rev., 2002, 102, 3589-3614.

7 S. Inagaki, S. Guan, T. Ohsuna and O. Terasaki, Nature, 2002, 416, 304-307.

8 A. Corma, H. García and F.X. Llabrés i Xamena, Chem. Rev., 2010, 110, 4606-4655.

9 E. Reale, A. Leyva, A. Corma, C. Martínez, H. García and F. Rey, J. Mater. Chem., 2005, 15, 1742-1754.

10 H. Li, M. Eddaoudi, M. O’Keeffe and O. M. Yaghi, Nature, 1999, 402, 276-279.

11 E. Ruíz-Hitzky, M. Darder and P. Aranda, J. Mater. Chem., 2005, 15, 3650-3662.

12 M.E. Leonowicz, J.A. Lawton, S.L. Lawton and M.K. Rubin, Science, 1994, 264, 1910-1913.

13 T.J. Pinnavaia, ACS Adv. Chem. Ser., 1995, 245, 283-300.

14 G. Lagaly, Solid State Ionics, 1986, 22, 43-51.

15 A. Corma, C. Corell, J. Pérez-Pariente, J.M. Guil, R. Guil-López, S. Nicolopoulos, J. González-Calbet and M. Vallet-Regí, Zeolites, 1996, 16, 7-14.

16 M. L. Occelli and R.M. Tindwa, Clays Clay Miner., 1983, 31, 22-28.

17 V. Srivastava, K. Gaubert, M. Pucheault and M. Vaultier, ChemCatChem, 2009, 1, 94-98.

18 K. Motokura, M. Tada and Y. Iwasawa, J. Am. Chem. Soc., 2009, 131, 7944-7945.

19 C. Baleizao, B. Gigante, M.J. Sabater, H. García and A. Corma, Appl. Catal. A: General, 2002, 228, 279288.

20 V. Ayala, A. Corma, M. Iglesias, J.A. Rincón and F. Sánchez, J. Catal., 2004, 224, 170-177.

21 A. Corma, V. Fornés and F. Rey, Adv. Mater., 2002, 14, 71-74. 
22 R. Ishii, T. Ikeda, T. Itoh, T. Ebina, T. Yokoyama, T. Hanaoka and F. Mizukami, J. Mater. Chem., 2006, $16,4035-4046$.

23 D. Mochizuki, S. Kowata and K. Kuroda, Chem. Mater., 2006, 18, 5223-5229.

24 A. Corma, U. Díaz, T. García, G. Sastre and A. Velty, J. Am. Chem. Soc., 2010, 132, 15011-15021.

25 N.R. Shiju, A.H. Alberts, S. Khalid, D.R. Brown and G. Rothenberg, Angew. Chem. Int. Ed., 2011, 50, 9615-9619.

26 S. Shylesh, A. Wagener, A. Seifert, S. Ernst and W.R. Thiel, Angew. Chem. Int. Ed., 2010, 49, 184-187.

27 M. Opanasenko, W.O. Parker, M. Shamzhy, E. Montanari, M. Bellettato, M. Mazur, R. Millini and J. Cejka, J. Am. Chem. Soc., 2014, 136, 2511-2519.

28 A. Corma, V. Fornés, S.B.C. Pergher, Th.L. Maesen and J.G. Buglass, Nature, 1998, 396, 353-356.

29 A. Corma, U. Díaz, M.E. Dómine and V. Fornés, J. Am. Chem. Soc., 2000, 122, 2804-2809.

30 C. González- Arellano, A. Corma, M. Iglesias and F. Sánchez, Adv. Synth. \& Catal., 2004, 346, 17581764.

31 A. Corma, E. Gutiérrez-Puebla, M. Iglesias, A. Monge, S. Pérez-Ferreras and F. Sánchez, Adv. Synth. \& Catal., 2006, 348, 1899-1907.

32 J.O. Barth, J. Kornatowski and J.A. Lercher, J. Mater. Chem., 2004, 12, 369-373.

33 J. Alauzun, A. Mehdi, R. Mouawia, C. Reyé and R. Corriu, J. Sol-Gel Sci. Technol., 2008, 46, 383-392.

34 J.J.E. Moreau, L. Vellutini, M. Wong Chi Man and C. Bied, J. Am. Chem. Soc., 2001, 123, 1509-1510.

35 J.J.E. Moreau, B.P.Pichon, M. Wong Chi Man, C. Bied, H. Pritzkow, J.L. Bantignies, P. Dieudonné and J. Sauvajol, Angew. Chem. Int. Ed., 2004, 43, 203-206.

36 G. Bellussi, R. Millini, E. Montanari, A. Carati, C. Rizzo, W.O.N. Parker, G. Cruciani, A. De Angelis, L. Bonoldi and S. Zanardi, Chem. Commun., 2012, 48, 7356-7358.

37 G. Bellussi, A. Carati, E. Di Paola, R. Millini, W.O.N. Parker Jr, C. Rizzo and S. Zanardi, Micropor. Mesopor. Mater., 2008, 113, 252-260.

38 G. Bellussi, A. Carati, C. Rizzo, U. Díaz Morales, S. Zanardi, W.O.N. Parker, R. Millini, World Patent, WO 2008/017513 A2, 2008.

39 S. Zanardi, G. Bellussi, A. Carati, E. Di Paola, R. Millini, W.O.N. Parker and C. Rizzo, Stud. Surf. Sci. Catal., 2008, 174B, 965-968.

40 G. Bellussi, E. Montanari, E. Di Paola, R. Millini, A. Carati, C. Rizzo, W.O.N. Parker Jr, M. Gemmi, E. Mugnaioli and S. Zanardi, Angew. Chem. Int. Ed., 2012, 51, 666-669.

41 S. Zanardi, W.O. Parker, A. Carati, G. Botti, E. Montanari, Micropor. Mesopor. Mater., 2013, 172, 200205. 
42 M. Belletato, L. Bonoldi, G. Cruciani, C. Flego, S. Guidetti, R. Millini, E. Montanari, W.O. Parker and S. Zanardi, J. Phys. Chem. C, 2014, 118, 7458-7467.

43 S.J. Gregg and K. S. W. Sing, Adsorption, Surface Area and Porosity, Academic Press, London, 1982, 111-190.

44 K.S.W. Sing, D.H. Everett, R.A.W. Haul, L. Moscou, R.A. Pierotti, J. Rouquerol and T. Siemieniewska, Pure Appl. Chem. 1985, 57, 603-619.

45 E.P. Barrett, L.G. Joyner and P.P. Halenda, J. Am. Chem. Soc. 1951, 73, 373-380.

46 J. S. Dailey and T. J. Pinnavaia, Chem. Mat., 1992, 4, 855-863.

47 W. J. Roth and D. L. Dorset, Micropor.Mesopor. Mater., 2011, 142, 32-36.

48 U. Brenn, H. Ernst, D. Freude, R. Herrmann, R. Jähnig, H. G. Karge, J. Kärger, T. König, B. Mädler, U. T. Pingel, D. Prochnow and W. Schwieger, Micropor. Mesopor. Mater., 2000, 40, 43-52.

49 R. A. Fletcher and D. M. Bibby, Clays Clay Miner., 1987, 35, 318-320.

50 D. Mochizuki, A. Shimojima, T. Imagawa and K. Kuroda, J. Am. Chem. Soc., 2005, 127, 7183-7191.

51 A. J. Blake, K. R. Franlin and B. M. Lowe, Dalton Trans., 1988, 2513-2517.

52 L. Schreyeck, P. Caullet, J.-C. Mougenel, J.-L. Guth and B. Marler, Chem. Commun., 1995, 2187-2188.

53 C. Yoshina-Ishii, T. Asefa, N. Coombs, M.J. MacLachlan and G.A. Ozin, Chem. Commun., 1999, 25392540.

54 D. Zhou, X.B. Luo, H.L. Zhang, C. Dong, Q.H. Xia, Z.M. Liu and F. Deng, Micropor. Mesopor. Mater., 2009, 121, 194-199.

55 E. Poli, E. Merino, U. Díaz, D. Brunel and A. Corma, J. Mater. Chem., 2011, 21, 8524-8527.

56 J.A. Bokhoven, A.L. Roest, D.C. Koningsberger, J.T. Miller, G.H. Nachtegaal and A.P.M. Kentgens, J. Phys. Chem. B, 2000, 104, 6743-6754.

57 L.J. Bellamy, Advances in infrared group frequencies, Chapman and Hall, London, 1968.

58 I. Rodriguez, S. Iborra, F. Rey and A. Corma, Appl. Catal. A, 2000, 194-195, $241-252$.

59 M. J. Climent, A. Corma, S. Iborra, K. Epping and A. Velty, J. Catal., 2004, 225, 316 -326.

60 F. S. Prout, U. D. Beaucaire, G. R. Dyrkarcz, W. M. Koppes, R. E. Kuznicki, T. A. Marlewski, J. A. Pienkowski and J. M. Puda, J. Org. Chem., 1973, 38, $1512-1517$.

61 G. Jones, Org. Reactions., 1967, 15, $204-599$.

62 J. Guyot and A. Kergomard, Tetrahedron, 1983, 39, 1167- 1179.

63 R. Chaudhuri, US Patent 0059258A1, 2007.

64 C. Wang, R. K . Chaudhuri, J. Jachowicz, B. C. Locke and F. M. Miksza, US Patent 5830441, 1998. 
65 L. Xu, C.G. Li, K. Zhang and P. Wu, ACS Catalysis, 2014, 4, 2959-2968.

66 L.A. Pine, P.J. Maher and W.A. Wachter, J. Catal., 1984, 85, 466-476. 